EUROPEAN ORGANISATION FOR NUCLEAR RESEARCH (CERN)

CERN-EP/2000-099

20th July 2000

\title{
Measurement of the Mass and Width of the $\mathrm{W}$ Boson in $\mathrm{e}^{+} \mathrm{e}^{-}$Collisions at $189 \mathrm{GeV}$
}

\author{
The OPAL Collaboration
}

\begin{abstract}
The mass and width of the $\mathrm{W}$ boson are determined in $\mathrm{e}^{+} \mathrm{e}^{-}$collisions at LEP using $183 \mathrm{pb}^{-1}$ of data recorded at a centre-of-mass energy $\sqrt{s}=189 \mathrm{GeV}$ with the OPAL detector. The invariant mass distributions from $970 \mathrm{~W}^{+} \mathrm{W}^{-} \rightarrow \mathrm{qqqq}$ and $1118 \mathrm{~W}^{+} \mathrm{W}^{-} \rightarrow \mathrm{qq} \ell \nu_{\ell}$ candidate events are used to measure the mass of the $\mathrm{W}$ boson,

$$
M_{\mathrm{W}}=80.451 \pm 0.076 \text { (stat.) } \pm 0.049 \text { (syst.) GeV. }
$$

A direct measurement of the width of the $\mathrm{W}$ boson gives $\Gamma_{\mathrm{W}}=2.09 \pm 0.18$ (stat.) \pm 0.09 (syst.) $\mathrm{GeV}$. The results are combined with previous OPAL results from $78 \mathrm{pb}^{-1}$ of data recorded with $\sqrt{s}$ from 161 to $183 \mathrm{GeV}$, to obtain:

$$
\begin{aligned}
M_{\mathrm{W}} & =80.432 \pm 0.066 \text { (stat.) } \pm 0.045 \text { (syst.) } \mathrm{GeV} \\
\Gamma_{\mathrm{W}} & =2.04 \pm 0.16 \text { (stat.) } \pm 0.09 \text { (syst.) GeV. }
\end{aligned}
$$

The consistency of the direct measurement of $M_{\mathrm{W}}$ with that inferred from other measurements of electroweak parameters provides an important test of the Standard Model of electroweak interactions.
\end{abstract}

(Submitted to Physics Letters B) 


\section{The OPAL Collaboration}

G. Abbiendi ${ }^{2}$, K. Ackerstaff ${ }^{8}$, C. Ainsley ${ }^{5}$, P.F. Åkesson ${ }^{3}$, G. Alexander ${ }^{22}$, J. Allison ${ }^{16}$, K.J. Anderson ${ }^{9}$, S. Arcelli ${ }^{17}$, S. Asai ${ }^{23}$, S.F. Ashby ${ }^{1}$, D. Axen ${ }^{27}$, G. Azuelos ${ }^{18, a}$, I. Bailey ${ }^{26}$, A.H. Ball ${ }^{8}$, E. Barberio ${ }^{8}$, R.J. Barlow ${ }^{16}$, S. Baumann ${ }^{3}$, T. Behnke ${ }^{25}$, K.W. Bell ${ }^{20}$, G. Bella ${ }^{22}$, A. Bellerive ${ }^{9}$, G. Benelli ${ }^{2}$, S. Bentvelsen ${ }^{8}$, S. Bethke ${ }^{32}$, O. Biebel ${ }^{32}$, I.J. Bloodworth ${ }^{1}$, O. Boeriu ${ }^{10}$, P. Bock ${ }^{11}$, J. Böhme ${ }^{14, h}$, D. Bonacorsi ${ }^{2}$, M. Boutemeur ${ }^{31}$, S. Braibant ${ }^{8}$, P. Bright-Thomas ${ }^{1}$, L. Brigliadori ${ }^{2}$,

R.M. Brown ${ }^{20}$, H.J. Burckhart ${ }^{8}$, J. Cammin ${ }^{3}$, P. Capiluppi ${ }^{2}$, R.K. Carnegie ${ }^{6}$, A.A. Carter ${ }^{13}$, J.R. Carter ${ }^{5}$, C.Y. Chang ${ }^{17}$, D.G. Charlton ${ }^{1, b}$, P.E.L. Clarke ${ }^{15}$, E. Clay ${ }^{15}$, I. Cohen ${ }^{22}$, O.C. Cooke ${ }^{8}$, J. Couchman ${ }^{15}$, C. Couyoumtzelis ${ }^{13}$, R.L. Coxe ${ }^{9}$, A. Csilling ${ }^{15, j}$, M. Cuffiani ${ }^{2}$, S. Dado ${ }^{21}$, G.M. Dallavalle ${ }^{2}$, S. Dallison ${ }^{16}$, A. de Roeck ${ }^{8}$, E. de Wolf ${ }^{8}$, P. Dervan ${ }^{15}$, K. $\operatorname{Desch}^{25}$, B. Dienes ${ }^{30, h}$, M.S. Dixit ${ }^{7}$, M. Donkers ${ }^{6}$, J. Dubbert ${ }^{31}$, E. Duchovni ${ }^{24}$, G. Duckeck ${ }^{31}$, I.P. Duerdoth ${ }^{16}$, P.G. Estabrooks ${ }^{6}$, E. Etzion ${ }^{22}$, F. Fabbri ${ }^{2}$, M. Fanti ${ }^{2}$, L. Feld ${ }^{10}$, P. Ferrari ${ }^{12}$, F. Fiedler ${ }^{8}$, I. Fleck ${ }^{10}$, M. Ford ${ }^{5}$, A. Frey ${ }^{8}$, A. Fürtjes ${ }^{8}$, D.I. Futyan ${ }^{16}$, P. Gagnon ${ }^{12}$, J.W. Gary ${ }^{4}$, G. Gaycken ${ }^{25}$,

C. Geich-Gimbel ${ }^{3}$, G. Giacomelli ${ }^{2}$, P. Giacomelli ${ }^{8}$, D. Glenzinski $^{9}$, J. Goldberg ${ }^{21}$, C. Grandi ${ }^{2}$, K. Graham ${ }^{26}$, E. Gross ${ }^{24}$, J. Grunhaus ${ }^{22}$, M. Gruwé2 ${ }^{25}$, P.O. Günther ${ }^{3}$, C. Hajdu ${ }^{29}$, G.G. Hanson ${ }^{12}$, M.Hansroul ${ }^{8}$, M. Hapke ${ }^{13}$, K. Harder ${ }^{25}$, A. Harel ${ }^{21}$, M. Harin-Dirac ${ }^{4}$, A. Hauke ${ }^{3}$, M. Hauschild ${ }^{8}$, C.M.Hawkes ${ }^{1}$, R.Hawkings ${ }^{8}$, R.J.Hemingway ${ }^{6}$, C. Hensel ${ }^{25}$, G.Herten ${ }^{10}$, R.D.Heuer ${ }^{25}$, J.C.Hill ${ }^{5}$,

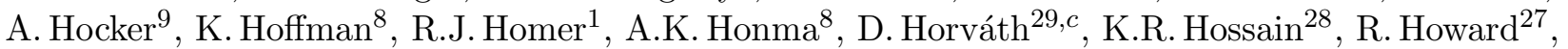

P. Hüntemeyer ${ }^{25}$, P. Igo-Kemenes ${ }^{11}$, K. Ishii ${ }^{23}$, F.R. Jacob ${ }^{20}$, A. Jawahery ${ }^{17}$, H. Jeremie ${ }^{18}$,

C.R. Jones ${ }^{5}$, P. Jovanovic ${ }^{1}$, T.R. Junk ${ }^{6}$, N. Kanaya ${ }^{23}$, J. Kanzaki ${ }^{23}$, G. Karapetian ${ }^{18}$, D. Karlen ${ }^{6}$, V. Kartvelishvili ${ }^{16}$, K. Kawagoe ${ }^{23}$, T. Kawamoto ${ }^{23}$, R.K. Keeler ${ }^{26}$, R.G. Kellogg ${ }^{17}$, B.W. Kennedy ${ }^{20}$, D.H. Kim ${ }^{19}$, K. Klein ${ }^{11}$, A. Klier ${ }^{24}$, S. Kluth ${ }^{32}$, T. Kobayashi' ${ }^{23}$, M. Kobel ${ }^{3}$, T.P. Kokott ${ }^{3}$,

S. Komamiya ${ }^{23}$, R.V.Kowalewski ${ }^{26}$, T. Kress ${ }^{4}$, P. Krieger ${ }^{6}$, J.von Krogh ${ }^{11}$, T. Kuhl ${ }^{3}$, M. Kupper ${ }^{24}$,

P. Kyberd ${ }^{13}$, G.D. Lafferty ${ }^{16}$, H. Landsman ${ }^{21}$, D. Lanske ${ }^{14}$, I. Lawson ${ }^{26}$, J.G. Layter ${ }^{4}$, A. Leins ${ }^{31}$,

D. Lellouch ${ }^{24}$, J. Letts ${ }^{12}$, L. Levinson ${ }^{24}$, R. Liebisch ${ }^{11}$, J. Lillich ${ }^{10}$, B. List ${ }^{8}$, C. Littlewood ${ }^{5}$,

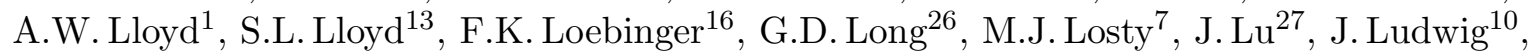
A. Macchiolo ${ }^{18}$, A. Macpherson ${ }^{28, m}$, W. Mader ${ }^{3}$, S. Marcellini' ${ }^{2}$, T.E. Marchant ${ }^{16}$, A.J. Martin ${ }^{13}$, J.P. Martin ${ }^{18}$, G. Martinez ${ }^{17}$, T. Mashimo ${ }^{23}$, P. Mättig ${ }^{24}$, W.J. McDonald ${ }^{28}$, J. McKenna ${ }^{27}$,

T.J. McMahon ${ }^{1}$, R.A. McPherson ${ }^{26}$, F. Meijers ${ }^{8}$, P. Mendez-Lorenzo ${ }^{31}$, W. Menges ${ }^{25}$, F.S. Merritt ${ }^{9}$,

H. Mes ${ }^{7}$, A. Michelini' ${ }^{2}$, S. Mihara ${ }^{23}$, G. Mikenberg ${ }^{24}$, D.J. Miller ${ }^{15}$, W. Mohr ${ }^{10}$, A. Montanari ${ }^{2}$, T. Mori ${ }^{23}$, K. Nagai ${ }^{8}$, I. Nakamura ${ }^{23}$, H.A. Neal ${ }^{12, f}$, R. Nisius ${ }^{8}$, S.W. O'Neale ${ }^{1}$, F.G. Oakham ${ }^{7}$, F. Odorici ${ }^{2}$, H.O. Ogren ${ }^{12}$, A. $\mathrm{Oh}^{8}$, A. Okpara ${ }^{11}$, M.J. Oreglia ${ }^{9}$, S. Orito ${ }^{23}$, G. Pásztor ${ }^{8, j}$, J.R. Pater ${ }^{16}$,

G.N. Patrick ${ }^{20}$, J.Patt ${ }^{10}$, P. Pfeifenschneider ${ }^{14, i}$, J.E. Pilcher ${ }^{9}$, J. Pinfold ${ }^{28}$, D.E. Plane ${ }^{8}$, B. Poli ${ }^{2}$,

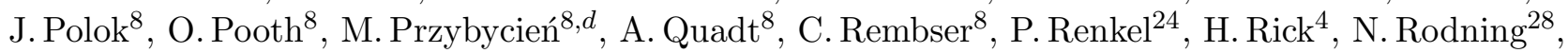

J.M. Roney ${ }^{26}$, S. Rosati ${ }^{3}$, K. Roscoe ${ }^{16}$, A.M. Rossi ${ }^{2}$, Y.Rozen ${ }^{21}$, K. Runge ${ }^{10}$, O. Runolfsson ${ }^{8}$, D.R. Rust ${ }^{12}$, K. Sachs ${ }^{6}$, T.Saeki ${ }^{23}$, O.Sahr ${ }^{31}$, E.K.G. Sarkisyan ${ }^{22}$, C. Sbarra ${ }^{26}$, A.D. Schaile ${ }^{31}$, O. Schaile ${ }^{31}$, P. Scharff-Hansen ${ }^{8}$, M. Schröder ${ }^{8}$, M. Schumacher ${ }^{25}$, C. Schwick ${ }^{8}$, W.G. Scott ${ }^{20}$, R. Seuster ${ }^{14, h}$, T.G. Shears ${ }^{8, k}$, B.C. Shen ${ }^{4}$, C.H. Shepherd-Themistocleous ${ }^{5}$, P. Sherwood ${ }^{15}$,

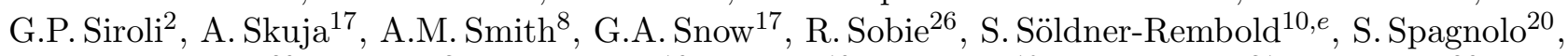

M. Sproston ${ }^{20}$, A. Stahl ${ }^{3}$, K. Stephens ${ }^{16}$, K. Stoll ${ }^{10}$, D. Strom ${ }^{19}$, R.Ströhmer ${ }^{31}$, L. Stumpf ${ }^{26}$,

B. Surrow ${ }^{8}$, S.D. Talbot ${ }^{1}$, S. Tarem ${ }^{21}$, R.J. Taylor ${ }^{15}$, R. Teuscher ${ }^{9}$, M. Thiergen ${ }^{10}$, J. Thomas ${ }^{15}$, M.A. Thomson ${ }^{8}$, E. Torrence ${ }^{9}$, S. Towers ${ }^{6}$, D. Toya ${ }^{23}$, T. Trefzger ${ }^{31}$, I. Trigger ${ }^{8}$, Z. Trócsányi ${ }^{30, g}$, E. Tsur ${ }^{22}$, M.F. Turner-Watson ${ }^{1}$, I. Ueda ${ }^{23}$, B. Vachon26, P. Vannerem ${ }^{10}$, M. Verzocchi ${ }^{8}$, H. Voss ${ }^{8}$,

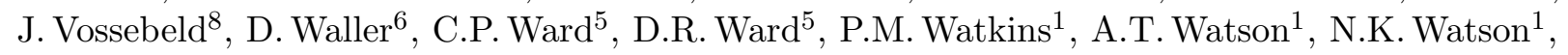
P.S. Wells ${ }^{8}$, T. Wengler ${ }^{8}$, N. Wermes ${ }^{3}$, D. Wetterling ${ }^{11}$ J.S. White $^{6}$, G.W.Wilson ${ }^{16}$, J.A. Wilson ${ }^{1}$, T.R. Wyatt $^{16}$, S. Yamashita ${ }^{23}$, V. Zacek ${ }^{18}$, D. Zer-Zion ${ }^{8, l}$

${ }^{1}$ School of Physics and Astronomy, University of Birmingham, Birmingham B15 2TT, UK 
${ }^{2}$ Dipartimento di Fisica dell' Università di Bologna and INFN, I-40126 Bologna, Italy

${ }^{3}$ Physikalisches Institut, Universität Bonn, D-53115 Bonn, Germany

${ }^{4}$ Department of Physics, University of California, Riverside CA 92521, USA

${ }^{5}$ Cavendish Laboratory, Cambridge CB3 0HE, UK

${ }^{6}$ Ottawa-Carleton Institute for Physics, Department of Physics, Carleton University, Ottawa, Ontario K1S 5B6, Canada

${ }^{7}$ Centre for Research in Particle Physics, Carleton University, Ottawa, Ontario K1S 5B6, Canada

${ }^{8}$ CERN, European Organisation for Nuclear Research, CH-1211 Geneva 23, Switzerland

${ }^{9}$ Enrico Fermi Institute and Department of Physics, University of Chicago, Chicago IL 60637, USA

${ }^{10}$ Fakultät für Physik, Albert Ludwigs Universität, D-79104 Freiburg, Germany

${ }^{11}$ Physikalisches Institut, Universität Heidelberg, D-69120 Heidelberg, Germany

${ }^{12}$ Indiana University, Department of Physics, Swain Hall West 117, Bloomington IN 47405, USA

${ }^{13}$ Queen Mary and Westfield College, University of London, London E1 4NS, UK

${ }^{14}$ Technische Hochschule Aachen, III Physikalisches Institut, Sommerfeldstrasse 26-28, D-52056 Aachen, Germany

${ }^{15}$ University College London, London WC1E 6BT, UK

${ }^{16}$ Department of Physics, Schuster Laboratory, The University, Manchester M13 9PL, UK

${ }^{17}$ Department of Physics, University of Maryland, College Park, MD 20742, USA

${ }^{18}$ Laboratoire de Physique Nucléaire, Université de Montréal, Montréal, Quebec H3C 3J7, Canada

${ }^{19}$ University of Oregon, Department of Physics, Eugene OR 97403, USA

${ }^{20}$ CLRC Rutherford Appleton Laboratory, Chilton, Didcot, Oxfordshire OX11 0QX, UK

${ }^{21}$ Department of Physics, Technion-Israel Institute of Technology, Haifa 32000, Israel

${ }^{22}$ Department of Physics and Astronomy, Tel Aviv University, Tel Aviv 69978, Israel

${ }^{23}$ International Centre for Elementary Particle Physics and Department of Physics, University of Tokyo, Tokyo 113-0033, and Kobe University, Kobe 657-8501, Japan

${ }^{24}$ Particle Physics Department, Weizmann Institute of Science, Rehovot 76100, Israel

${ }^{25}$ Universität Hamburg/DESY, II Institut für Experimental Physik, Notkestrasse 85, D-22607 Hamburg, Germany

${ }^{26}$ University of Victoria, Department of Physics, P O Box 3055, Victoria BC V8W 3P6, Canada

${ }^{27}$ University of British Columbia, Department of Physics, Vancouver BC V6T 1Z1, Canada

${ }^{28}$ University of Alberta, Department of Physics, Edmonton AB T6G 2J1, Canada

${ }^{29}$ Research Institute for Particle and Nuclear Physics, H-1525 Budapest, P O Box 49, Hungary

${ }^{30}$ Institute of Nuclear Research, H-4001 Debrecen, P O Box 51, Hungary

${ }^{31}$ Ludwigs-Maximilians-Universität München, Sektion Physik, Am Coulombwall 1, D-85748 Garching, Germany

${ }^{32}$ Max-Planck-Institute für Physik, Föhring Ring 6, 80805 München, Germany

${ }^{a}$ and at TRIUMF, Vancouver, Canada V6T 2A3

${ }^{b}$ and Royal Society University Research Fellow

${ }^{c}$ and Institute of Nuclear Research, Debrecen, Hungary

${ }^{d}$ and University of Mining and Metallurgy, Cracow

$e$ and Heisenberg Fellow

${ }^{f}$ now at Yale University, Dept of Physics, New Haven, USA

$g$ and Department of Experimental Physics, Lajos Kossuth University, Debrecen, Hungary

$h$ and MPI München

${ }^{i}$ now at MPI für Physik, 80805 München

$j$ and Research Institute for Particle and Nuclear Physics, Budapest, Hungary

${ }^{k}$ now at University of Liverpool, Dept of Physics, Liverpool L69 3BX, UK

${ }^{l}$ and University of California, Riverside, High Energy Physics Group, CA 92521, USA

$m$ and CERN, EP Div, 1211 Geneva 23. 


\section{Introduction}

The LEP $\mathrm{e}^{+} \mathrm{e}^{-}$collider at CERN provides an ideal environment for the study of the properties of the gauge bosons of the Standard Model (SM) of electroweak interactions [1]. In the first stage of its operation, LEP produced $\mathrm{e}^{+} \mathrm{e}^{-}$collisions at centre-of-mass energies, $\sqrt{s}$, within a few $\mathrm{GeV}$ of the $\mathrm{Z}^{0}$ resonance, allowing precise measurements of the properties of the $\mathrm{Z}^{0}$ boson including its mass and fermionic couplings [2, 3]. In the context of the SM these measurements place constraints on the mass of the Higgs boson and provide an indirect determination of the mass of the $\mathrm{W}$ boson, $M_{\mathrm{W}}$. Since 1996, the LEP collider has operated above the threshold for $\mathrm{W}^{+} \mathrm{W}^{-}$production (LEP2), allowing measurements of the trilinear gauge boson couplings [4] and a direct measurement of $M_{\mathrm{W}}$. When combined with the direct measurements of the top quark mass at the Tevatron [5], measurements of $M_{\mathrm{W}}$ enable further constraints to be set on the mass of the Higgs boson via electroweak radiative corrections [6, [7]. Comparison between the direct measurements of the mass of the $\mathrm{W}$ boson and the value determined indirectly from data recorded at $\sqrt{s} \approx M_{\mathrm{Z}}$ provides an important test of the self-consistency of the Standard Model. The direct measurement of $\Gamma_{\mathrm{W}}$ further tests the consistency of the Standard Model.

The combination of direct measurements of $M_{\mathrm{W}}$ from LEP2 at $\sqrt{s} \sim 161-183 \mathrm{GeV}$ [8] 13 ] and from hadron colliders [14] currently give $M_{\mathrm{W}}=80.419 \pm 0.056 \mathrm{GeV}$ [15]. This direct measurement is consistent with the indirect value obtained from lower energy data, primarily measurements at $\sqrt{s} \sim M_{\mathrm{Z}}$, which give $M_{\mathrm{W}}^{\text {indirect }}=80.382 \pm 0.026 \mathrm{GeV}$ [3].

The published OPAL measurements of $M_{\mathrm{W}}$ are based on approximately $78 \mathrm{pb}^{-1}$ of data. This paper describes a measurement of the mass of the $\mathrm{W}$ boson using a further $183 \mathrm{pb}^{-1}$ of data recorded by OPAL during 1998 at $\sqrt{s} \sim 189 \mathrm{GeV}$. This result is combined with previous OPAL measurements to give a direct measurement of the mass of the $\mathrm{W}$ boson with a total uncertainty of $79 \mathrm{MeV}$. It is expected that the ultimate LEP precision on $M_{\mathrm{W}}$ will be approximately $30 \mathrm{MeV}$ when all data are included and the results of the four LEP experiments are combined [16].

\section{Data and Monte Carlo Samples}

A detailed description of the OPAL detector can be found in 17]. The data sample used for this analysis corresponds to an accepted integrated luminosity, evaluated using small angle Bhabha scattering events observed in the forward calorimeters [18], of $183.1 \pm 0.2$ (stat.) \pm 0.4 (syst.) $\mathrm{pb}^{-1}$. The luminosity weighted mean centre-of-mass energy for the data sample is $\sqrt{s}=188.635 \pm 0.040 \mathrm{GeV}$ [19].

\subsection{Monte Carlo samples}

A number of Monte Carlo generators are used to simulate the physics processes relevant to the studies presented in this paper. All samples include a full simulation of the OPAL detector [20]. The main physics processes at LEP2 can be broken down into three main categories: four-fermion $(4 f)$ production, including $\mathrm{e}^{+} \mathrm{e}^{-} \rightarrow \mathrm{W}^{+} \mathrm{W}^{-} \rightarrow 4 f$ and $\mathrm{e}^{+} \mathrm{e}^{-} \rightarrow \mathrm{Z}^{0} \mathrm{Z}^{0} \rightarrow 4 f$, but excluding contributions from multi-peripheral diagrams; two-fermion production; and multi-peripheral two-photon mediated processes. For the measurement of the $\mathrm{W}$ boson mass and width, only the four-fermion processes and the two-fermion background process $\mathrm{e}^{+} \mathrm{e}^{-} \rightarrow \mathrm{Z}^{0} / \gamma \rightarrow \mathrm{q} \overline{\mathrm{q}}$ play an important role.

The Koralw program [21], which uses matrix elements calculated with grc4f [22], is used to 
simulate the production of most four-fermion final states, $\mathrm{e}^{+} \mathrm{e}^{-} \rightarrow 4 f$. The main Monte Carlo samples are generated at $\sqrt{s}=188.634 \mathrm{GeV}$ with $M_{\mathrm{W}}=80.33 \mathrm{GeV}$. The samples were generated using the running width scheme for the Breit-Wigner distribution. The four-fermion samples are divided into final states which have contributions from processes involving the W-boson propagator and those which do not. In the invariant mass region close to $M_{\mathrm{W}}$, the four-fermion cross-section is dominated by doubly resonant W-pair production diagrams (CC03)円. Additional KorALW four-fermion Monte Carlo samples are produced with different centre-of-mass energies and with different values of $M_{\mathrm{W}}$. The four-fermion background from the process $\mathrm{e}^{+} \mathrm{e}^{-} \rightarrow \mathrm{e}^{+} \mathrm{e}^{-} \mathrm{q} \overline{\mathrm{q}}$ is simulated using the grc4f [22] generator. The most important two-fermion background process, $\mathrm{e}^{+} \mathrm{e}^{-} \rightarrow \mathrm{Z}^{0} / \gamma \rightarrow \mathrm{q} \overline{\mathrm{q}}$, is simulated using Pythia [23], with HERWIG [24] used to assess possible systematic uncertainties.

\section{$3 \quad \mathbf{W}^{+} \mathbf{W}^{-}$Event Selection}

The event selections are described in [25] and references therein. The selections are sensitive to the leptonic $\mathrm{W}^{+} \mathrm{W}^{-} \rightarrow \ell \nu_{\ell} \ell \nu_{\ell}$, semi-leptonic $\mathrm{W}^{+} \mathrm{W}^{-} \rightarrow \mathrm{qq} \ell \nu_{\ell}$, and hadronic $\mathrm{W}^{+} \mathrm{W}^{-} \rightarrow \mathrm{qqqq}$ final states. Due to the presence of two unobserved prompt neutrinos in the $\mathrm{W}^{+} \mathrm{W}^{-} \rightarrow \ell \nu_{\ell} \ell \nu_{\ell}$ final state there is little sensitivity to $M_{\mathrm{W}}$ and the leptonic final state is therefore not used here.

Semi-leptonic $\mathrm{W}^{+} \mathrm{W}^{-} \rightarrow \mathrm{qq} \ell \nu_{\ell}$ decays comprise $44 \%$ of the total $\mathrm{W}^{+} \mathrm{W}^{-}$cross-section. The event selection employs three multivariate relative likelihood discriminants, one for each of the $\mathrm{W}^{+} \mathrm{W}^{-} \rightarrow$ $\mathrm{qqe} \nu_{\mathrm{e}}, \mathrm{W}^{+} \mathrm{W}^{-} \rightarrow \mathrm{qq} \mu \nu_{\mu}$, and $\mathrm{W}^{+} \mathrm{W}^{-} \rightarrow \mathrm{qq} \tau \nu_{\tau}$ final states. The $\mathrm{W}^{+} \mathrm{W}^{-} \rightarrow \mathrm{qqe} \nu_{\mathrm{e}}$ and $\mathrm{W}^{+} \mathrm{W}^{-} \rightarrow$ $\mathrm{qq} \mu \nu_{\mu}$ channels are characterised by two well-separated hadronic jets, a high-momentum lepton and missing momentum due to the prompt neutrino from the leptonic $\mathrm{W}$ decay. The signature for the $\mathrm{W}^{+} \mathrm{W}^{-} \rightarrow \mathrm{qq} \tau \nu_{\tau}$ channel is similar, with the exception that the $\tau$ lepton is identified as an isolated, low-multiplicity jet typically consisting of one or three tracks. $\mathrm{W}^{+} \mathrm{W}^{-} \rightarrow \mathrm{qq} \ell \nu_{\ell}$ events are selected with an efficiency of $87 \%$ and a purity of $91 \%$. The dominant backgrounds are $\mathrm{Z}^{0} / \gamma \rightarrow \mathrm{q} \overline{\mathrm{q}}$ and four-fermion processes such as $\mathrm{e}^{+} \mathrm{e}^{-} \rightarrow \mathrm{We} \nu_{\mathrm{e}}$ and $\mathrm{e}^{+} \mathrm{e}^{-} \rightarrow\left(\mathrm{Z}^{0} / \gamma\right)^{*}\left(\mathrm{Z}^{0} / \gamma\right)^{*} \rightarrow \mathrm{q} \overline{\mathrm{q}} \ell^{+} \ell^{-}$.

Hadronic $\mathrm{W}^{+} \mathrm{W}^{-} \rightarrow$ qqqq decays comprise $46 \%$ of the total $\mathrm{W}^{+} \mathrm{W}^{-}$cross-section and are identified by requiring four energetic hadronic jets and little or no missing energy. A preselection removes approximately $98 \%$ of the dominant background process, $\mathrm{Z}^{0} / \gamma \rightarrow \mathrm{q} \overline{\mathrm{q}}$. A multivariate relative likelihood discriminant is then employed to select the $\mathrm{W}^{+} \mathrm{W}^{-} \rightarrow$ qqqq candidates with an efficiency of $87 \%$ and a purity of $77 \%$.

After the selections are applied, $1546 \mathrm{~W}^{+} \mathrm{W}^{-} \rightarrow$ qqqq and $1246 \mathrm{~W}^{+} \mathrm{W}^{-} \rightarrow \mathrm{qq} \ell \nu_{\ell}$ candidate events remain, consistent with Standard Model expectations. Not all events are used in the measurements described here. As discussed in Section 4.1, additional cuts are applied to remove poorly reconstructed events and further reduce backgrounds.

\section{Measurement of the Mass and Width of the W Boson}

The measurement of the mass and width of the $\mathrm{W}$ boson proceeds in two stages. Firstly the invariant masses of the $\mathrm{W}$ decay products are reconstructed on an event-by-event basis. Kinematic fits are applied to each selected $\mathrm{W}^{+} \mathrm{W}^{-} \rightarrow \mathrm{qq} \ell \nu_{\ell}$ and $\mathrm{W}^{+} \mathrm{W}^{-} \rightarrow$ qqqq event to improve the mass resolution. The reconstructed invariant mass spectra are then used to determine $M_{\mathrm{W}}$ and $\Gamma_{\mathrm{W}}$.

\footnotetext{
${ }^{1}$ In this paper, the doubly-resonant $\mathrm{W}$ pair production diagrams, i.e. $t$-channel $\nu_{\mathrm{e}}$ exchange and $s$-channel $\mathrm{Z}^{0} / \gamma$ exchange, are referred to as "CC03", following the notation of [16].
} 
Fits to the invariant mass spectra to obtain $M_{\mathrm{W}}$ are performed using three different techniques. The central results of this paper are obtained using a Monte Carlo reweighting technique [16 to fit the observed mass spectra to obtain $M_{\mathrm{W}}$ and $\Gamma_{\mathrm{W}}$. The $\mathrm{W}$ mass is also determined using two alternative methods, which are used as cross-checks. In the first, an analytic fit to the measured mass spectrum uses an unbinned likelihood fit method to determine $M_{\mathrm{W}}$. To describe the signal shape, the fit uses a parametrisation based on a Breit-Wigner function [9]. The second method uses a convolution technique [12, 16]. The three different fitting techniques have similar expected statistical sensitivities and similar estimated systematic uncertainties.

\subsection{Invariant mass reconstruction}

The three methods for extracting $M_{\mathrm{W}}$ use nearly identical procedures to reconstruct the invariant mass of the $\mathrm{W}$ candidates. The description here applies to the reweighting method. Small variations relevant for the alternative analyses are discussed in Section 4.3 .

In previous OPAL measurements of $M_{\mathrm{W}}$ the tracks and clusters in selected $\mathrm{W}^{+} \mathrm{W}^{-} \rightarrow$ qqqq events were grouped into four jets using the Durham algorithm [26]. For the results presented here, $\mathrm{W}^{+} \mathrm{W}^{-} \rightarrow$ qqqq events are grouped into either four or five jet topologies depending on the value of $\ln \left(y_{45}\right)$, where $y_{45}$ is the value of the Durham jet resolution parameter at which the transition from 5 to 4 jets occurs. Events with $\ln \left(y_{45}\right)>-5.6$ are treated as five jets. This separation allows for the possibility of hard gluon radiation from one of the quarks and is found to improve the expected statistical sensitivity to $M_{\mathrm{W}}$ by approximately $5 \%$ compared with treating all events as four jets. The improvement in statistical sensitivity comes from events where, under the four jet hypothesis, a gluon jet from one $\mathrm{W}$ boson is combined with a quark jet from a different $\mathrm{W}$ boson. In the semi-leptonic decay channels, $\mathrm{W}^{+} \mathrm{W}^{-} \rightarrow \mathrm{qq} \ell \nu_{\ell}$, the lepton candidate is removed and the hadronic part of the event is reconstructed as two jets. The division of $\mathrm{W}^{+} \mathrm{W}^{-} \rightarrow \mathrm{qq} \ell \nu_{\ell}$ events into two and three jet would not improve the mass resolution. After the association of tracks and clusters into jets, corrections derived from the Monte Carlo simulation are applied to the measured jet momenta to account for double counting from particles which deposit energy in more than one sub-detector [27].

The invariant masses of the two $\mathrm{W}$ bosons can be determined directly from the reconstructed momenta of the observed decay products. However, the mass resolution is limited by the relatively large uncertainty on the measured energies of the jets, $\sigma_{E} / E \approx 20 \%$, rather than by uncertainties on the measured jet directions. For this reason the use of a kinematic fit which imposes the four constraints of energy and momentum conservation [28] (4-C fit) significantly improves the invariant mass resolution. The improvements mainly arise from the energy in event being constrained to the well measured centre-of-mass energy. The output of the 4-C fit consists of two reconstructed masses per event, one for each $\mathrm{W}$ boson in the final state. The resolution of the kinematic fit can be improved by imposing a further constraint that the masses of the two reconstructed $\mathrm{W}$ boson candidates are equal (5-C fit), yielding a single reconstructed mass per event. For $\mathrm{W}^{+} \mathrm{W}^{-} \rightarrow \mathrm{qq} \ell \nu_{\ell}$ events the three unmeasured variables corresponding to the neutrino momentum means that the effective number of constraints in the semi-leptonic is two, giving a 2-C fit. For the results presented in this paper, the mass reconstruction is performed using the 5 -C fit for $\mathrm{W}^{+} \mathrm{W}^{-} \rightarrow$ qqqq events and the 2-C fit for $\mathrm{W}^{+} \mathrm{W}^{-} \rightarrow \mathrm{qq} \ell \nu_{\ell}$ events.

A common kinematic fitting algorithm is used for $\mathrm{W}^{+} \mathrm{W}^{-} \rightarrow \mathrm{qqe} \nu_{\mathrm{e}}, \mathrm{W}^{+} \mathrm{W}^{-} \rightarrow \mathrm{qq} \mu \nu_{\mu}$ and $\mathrm{W}^{+} \mathrm{W}^{-} \rightarrow$ qqqq events. The fitted mass is obtained using an iterative $\chi^{2}$-minimisation procedure where the constraints in the kinematic fit are implemented using Lagrange multipliers. The presence of initial state radiation (ISR) is neglected. The corrected jet momenta, their associated errors, and the measured jet masses are input to the kinematic fit. The errors on the measured jet momenta 
are parameterised by expressions derived from Monte Carlo studies, which are functions of the visible energy and polar angle of the jet. The jet masses are fixed to their measured values. This is found to improve the mass resolution from the fit compared to treating jets as massless and also to reduce the bias in the fitted mass distribution.

The mass reconstruction for $\mathrm{W}^{+} \mathrm{W}^{-} \rightarrow \mathrm{qq} \tau \nu_{\tau}$ events is different. In the $\mathrm{W}^{+} \mathrm{W}^{-} \rightarrow \mathrm{qq} \tau \nu_{\tau}$ system, the absence of a measurement of the tau lepton energy means that the $\mathrm{W}$ mass information is determined entirely by the hadronic system. The absence of a measurement of the tau lepton energy reduces the effective number of constraints in the kinematic to one. Previously $[10$ the reconstructed mass in $\mathrm{W}^{+} \mathrm{W}^{-} \rightarrow \mathrm{qq} \tau \nu_{\tau}$ events was obtained from the invariant mass of the jet-jet system, scaled by the ratio of the beam energy to the sum of the jet energies. For the results presented here an analytic formula which reproduces the results of the 1-C kinematic fit is used.

For $\mathrm{W}^{+} \mathrm{W}^{-} \rightarrow \mathrm{qqe} \nu_{\mathrm{e}}$ and $\mathrm{W}^{+} \mathrm{W}^{-} \rightarrow \mathrm{qq} \mu \nu_{\mu}$ events the lepton direction is taken to coincide with the direction of the track associated with the electron or muon candidate. The energy is estimated from the associated electromagnetic calorimeter cluster for electrons and from the momentum of the track for muons. Unassociated electromagnetic clusters close to the lepton track, consistent with being from final state radiation (FSR), are included in the energy calculation. For each event a single mass is determined from a 2-C kinematic fit. In addition to the fitted mass, $m_{\mathrm{rec}}$, the error on the fitted mass, $\sigma_{\text {rec }}$, and the $\chi^{2}$ fit probability, $P_{\text {FIT }}$, are calculated. Events with $m_{\text {rec }}>65 \mathrm{GeV}$ and $P_{\text {FIT }}>0.001$ are retained. About half of the $\mathrm{W}^{+} \mathrm{W}^{-} \rightarrow \mathrm{qq} \tau \nu_{\tau}$ events selected as either $\mathrm{W}^{+} \mathrm{W}^{-} \rightarrow \mathrm{qqe} \nu_{\mathrm{e}}$ or $\mathrm{W}^{+} \mathrm{W}^{-} \rightarrow \mathrm{qq} \mu \nu_{\mu}$ fail these. The mass information is recovered by treating events with $m_{\text {rec }}<65 \mathrm{GeV}$ or $P_{\text {FIT }}<0.001$ as $\mathrm{W}^{+} \mathrm{W}^{-} \rightarrow \mathrm{qq} \tau \nu_{\tau}$ events. In addition, about $4 \%$ of selected $\mathrm{W}^{+} \mathrm{W}^{-} \rightarrow \mathrm{qqe} \nu_{\mathrm{e}}$ and $\mathrm{W}^{+} \mathrm{W}^{-} \rightarrow \mathrm{qq} \mu \nu_{\mu}$ events have the identified lepton beyond the effective tracking acceptance of the OPAL detector, within $20^{\circ}$ of the beam axis, in which case the lepton energy is either poorly measured or not measured at all. These events are included in the $\mathrm{W}^{+} \mathrm{W}^{-} \rightarrow \mathrm{qq} \tau \nu_{\tau}$ channel. The numbers of events used in each of the $\mathrm{W}^{+} \mathrm{W}^{-} \rightarrow \mathrm{qq} \ell \nu_{\ell}$ channels are given in Table 1 .

The situation for $\mathrm{W}^{+} \mathrm{W}^{-} \rightarrow$ qqqq events is complicated by the fact that there are three possible assignments of four jets to the two $\mathrm{W}$ bosons. For events reconstructed as five jets there are ten possible assignments of the jets to the $\mathrm{W}$ bosons. Incorrect combinations contain little or no information on the mass of the $\mathrm{W}$ boson. The incorrect jet-pairings result in a combinatorial background. For each $\mathrm{W}^{+} \mathrm{W}^{-} \rightarrow$ qqqq event, three (or ten) kinematic fits are performed, corresponding to the possible jetpairings. To eliminate poorly reconstructed events and reduce backgrounds, only combinations which give a successful 5-C kinematic fit with a resulting $\chi^{2}$ fit probability $P_{\text {FIT }}>0.01$ and $m_{\text {rec }}>65 \mathrm{GeV}$ are considered. A multivariate relative likelihood discriminant similar to that described in [10] is employed to pick out a single combination for each event and reduce combinatorial background. Different variables are used for the jet-pairing likelihoods for the four and five jet cases. For four jet events, the combination used in the fit is selected on the basis of two variables: the difference between the two fitted masses from the 4-C fit and the sum of the di-jet opening angles. In the five jet sample four variables are used: the 5-C fit mass, the difference between the two fitted masses from the 4-C fit, the minimum opening angle between the jets in the system assigned as $\mathrm{W}^{ \pm} \rightarrow \mathrm{q} \overline{\mathrm{q}} g$ and the cosine of the polar angle of the reconstructed $\mathrm{W}^{ \pm} \rightarrow \mathrm{q} \overline{\mathrm{q}} g$ system. For events reconstructed as four (five) jets, the combination corresponding to the largest jet-pairing likelihood is retained provided it has a likelihood output exceeding $0.40(0.42)$. Monte Carlo studies indicate that in $89 \%(70 \%)$ of the surviving signal events, the selected combination corresponds to the correct jet-pairing. The number of surviving events in the $\mathrm{W}^{+} \mathrm{W}^{-} \rightarrow$ qqqq channel is given in Table 1. Figure 1 1 shows the reconstructed mass distributions in both four and five jet channels before and after the jet-pairing likelihood cuts.

For events without ISR the average $m_{\text {rec }}$ resolution (as defined in [10]) for the correct jet pairing in $\mathrm{W}^{+} \mathrm{W}^{-} \rightarrow$ qqqq events is $1.7 \mathrm{GeV}$. For $\mathrm{W}^{+} \mathrm{W}^{-} \rightarrow$ qq $\ell \nu_{\ell}$ events the average $m_{\text {rec }}$ resolution is 
$2.4 \mathrm{GeV}, 2.8 \mathrm{GeV}$ and $3.4 \mathrm{GeV}$ in the $\mathrm{W}^{+} \mathrm{W}^{-} \rightarrow \mathrm{qqe} \nu_{\mathrm{e}}, \mathrm{W}^{+} \mathrm{W}^{-} \rightarrow \mathrm{qq} \mu \nu_{\mu}$ and $\mathrm{W}^{+} \mathrm{W}^{-} \rightarrow \mathrm{qq} \tau \nu_{\tau}$ channels respectively.

\subsection{Extraction of the $\mathrm{W}$ mass and width}

The Monte Carlo reweighting technique is used to provide the central results of this paper. The W boson mass and width are measured by directly comparing the reconstructed mass spectra in the data to Monte Carlo mass spectra corresponding to different values of $M_{\mathrm{W}}$ and $\Gamma_{\mathrm{W}}$. A likelihood fit is then used to extract $M_{\mathrm{W}}$ and $\Gamma_{\mathrm{W}}$ by determining which Monte Carlo spectrum best describes the data. The Monte Carlo spectra for arbitrary $\left(M_{\mathrm{W}}, \Gamma_{\mathrm{W}}\right)$ are obtained using the reweighting technique described in [9]. In previous OPAL publications, $\mathrm{W}^{+} \mathrm{W}^{-}$Monte Carlo samples generated using only CC03 diagrams were reweighted using the ratio of Breit-Wigner functions. For this analysis, KorALW $\mathrm{e}^{+} \mathrm{e}^{-} \rightarrow 4 f$ reference samples generated with $\left(M_{\mathrm{W}}=80.33 \mathrm{GeV}, \Gamma_{\mathrm{W}}=2.093 \mathrm{GeV}\right)$ are reweighted to $\left(M_{\mathrm{W}}^{\prime}, \Gamma_{\mathrm{W}}^{\prime}\right)$ using the ratio of Breit-Wigner functions. The reference samples used for the reweighting include only final states which have contributions from diagrams involving the $\mathrm{W}$ propagator. This procedure is found to give a good approximation to the more exact treatment of using the full fourfermion matrix elements, introducing a bias of less than $5 \mathrm{MeV}$ in the $\mathrm{W}^{+} \mathrm{W}^{-} \rightarrow \mathrm{qqe} \nu_{\mathrm{e}}$ channel and less than $2 \mathrm{MeV}$ in the other channels. Using the ratio of Breit-Wigner functions rather than four-fermion matrix elements results in a much faster fit.

The mass spectra for background events are taken from Monte Carlo and are assumed to be independent of $M_{\mathrm{W}}$ and $\Gamma_{\mathrm{W}}$. The main sources of background are $\mathrm{e}^{+} \mathrm{e}^{-} \rightarrow \mathrm{Z}^{0} / \gamma \rightarrow \mathrm{q} \overline{\mathrm{q}}$ and fourfermion processes. The background mass distributions are normalised to the expected number of background events. The reweighted signal spectra are then normalised such that the total number of signal plus background events corresponds to the observed number of events. This is done separately for the $\mathrm{W}^{+} \mathrm{W}^{-} \rightarrow \mathrm{qqqq}, \mathrm{W}^{+} \mathrm{W}^{-} \rightarrow \mathrm{qqe} \nu_{\mathrm{e}}, \mathrm{W}^{+} \mathrm{W}^{-} \rightarrow \mathrm{qq} \mu \nu_{\mu}$ and $\mathrm{W}^{+} \mathrm{W}^{-} \rightarrow \mathrm{qq} \tau \nu_{\tau}$ channels, with the $\mathrm{W}^{+} \mathrm{W}^{-} \rightarrow$ qqqq channel split into four and five jet topologies. In addition, the $\mathrm{W}^{+} \mathrm{W}^{-} \rightarrow \mathrm{qq} \ell \nu_{\ell}$ channels are divided into four subsamples according to the error on the reconstructed invariant mass, $\sigma_{\text {rec}}$. This division gives a larger weight to events with reconstructed masses which are known with better precision (i.e. small $\sigma_{\text {rec }}$ ) and reduces the expected statistical uncertainty on the fitted W mass from the $\mathrm{W}^{+} \mathrm{W}^{-} \rightarrow \mathrm{qq} \ell \nu_{\ell}$ channels by approximately $5 \%$. In the $\mathrm{W}^{+} \mathrm{W}^{-} \rightarrow$ qqqq channels, the width of the reconstructed mass distribution is dominated by the intrinsic width of the W. Consequently a similar subdivision into bins of $\sigma_{\text {rec }}$ does not improve the $M_{\mathrm{W}}$ sensitivity. However, jet-pairings which give a large jet-pairing likelihood are more likely to be correct and have a better mass resolution as shown in Figure 2. For this reason, the $\mathrm{W}^{+} \mathrm{W}^{-} \rightarrow$ qqqq events reconstructed as four jets are subdivided into four bins of jet-pairing likelihood, resulting in a $7 \%$ improvement in the statistical sensitivity to $M_{\mathrm{W}}$. No subdivision is performed for the five jet events.

A binned log-likelihood fit to the $m_{\text {rec }}$ distributions of the data is performed in the range $m_{\text {rec }}>$ $65 \mathrm{GeV}$. The log-likelihood function is identical to that used previously [10]. A log-likelihood curve is determined separately for each channel. For the $\mathrm{W}^{+} \mathrm{W}^{-} \rightarrow \mathrm{qq} \ell \nu_{\ell}$ channels, the results are obtained by adding the log-likelihood curves separately determined from each channel in each bin of $\sigma_{\text {rec }}$. For the $\mathrm{W}^{+} \mathrm{W}^{-} \rightarrow$ qqqq channel, the results are obtained by adding the likelihood curves obtained from the five jet events to the likelihood curves obtained from the four bins of jet-pairing likelihood for events reconstructed as four jets.

Two types of fit are performed. In the one parameter fit, $\Gamma_{\mathrm{W}}$ is constrained by the SM relation [16],

$$
\Gamma_{\mathrm{W}}=3 G_{\mathrm{F}} M_{\mathrm{W}}^{3}\left(1+2 \alpha_{\mathrm{S}} / 3 \pi\right) /(2 \sqrt{2} \pi),
$$


and only $M_{\mathrm{W}}$ is determined. The results of this fit for each channel are given in Table 2 and displayed in Figure 3. The combined result is discussed in Section 6. In the two parameter fit, both $M_{\mathrm{W}}$ and $\Gamma_{\mathrm{W}}$ are determined simultaneously.

In the reweighting method the fitted parameters are expected to be unbiased since any offsets in the reconstructed mass introduced in the analysis are implicitly accounted for in the Monte Carlo reconstructed mass spectra used in the reweighting procedure. This is verified using several Monte Carlo samples generated at various $M_{\mathrm{W}}$ and $\Gamma_{\mathrm{W}}$. In addition, tests using a large ensemble of Monte Carlo subsamples, each corresponding to $183 \mathrm{pb}^{-1}$ and including background contributions, are used to verify for each channel separately and for all channels combined, that the measured fit errors accurately reflect the root-mean-squared spread of the residual distribution for both the $M_{\mathrm{W}}$ and $\Gamma_{\mathrm{W}}$ fits. Since $M_{\mathrm{W}}$ in the Monte Carlo corresponds to the running width definition, so does the fitted mass. The expected statistical error on the $\mathrm{W}$ mass from the combination of the qq $\ell \nu_{\ell}$ and qqqq channels is $76 \pm 1 \mathrm{MeV}$, where the weights given to the two channels are determined by the both statistical and systematic error contributions. The quoted uncertainty on the expected statistical error is from Monte Carlo statistics.

\subsection{Alternative fit methods}

\subsubsection{Breit-Wigner fit}

The Breit-Wigner method is analogous to that described in [10]. It employs an unbinned maximumlikelihood fit to the reconstructed mass spectrum using an analytic Breit-Wigner function to describe the mass spectrum from $\mathrm{e}^{+} \mathrm{e}^{-} \rightarrow \mathrm{W}^{+} \mathrm{W}^{-}$. Due to initial-state radiation, the reconstructed mass spectrum is asymmetric. For the $\mathrm{W}^{+} \mathrm{W}^{-} \rightarrow \mathrm{qq} \ell \nu_{\ell}$ channels a relativistic Breit-Wigner function, with different widths above and below the peak, gives a satisfactory description of the $m_{\text {rec }}$ lineshape. The fitting function used is

$$
S\left(m_{\mathrm{rec}}\right)=A \frac{m_{\mathrm{rec}}^{2} \Gamma_{+(-)}^{2}}{\left(m_{\mathrm{rec}}^{2}-m_{0}^{2}\right)^{2}+m_{\mathrm{rec}}^{2} \Gamma_{+(-)}^{2}},
$$

where $\Gamma_{+(-)}$is the width assumed for all $m_{\text {rec }}$ above (below) the peak centred at $m_{0}$. This empirical choice of fitting function provides an adequate description for samples up to ten times the integrated luminosity of the data. The widths, $\Gamma_{+(-)}$, are fixed to values determined from fits to $\mathrm{W}^{+} \mathrm{W}^{-}$signal Monte Carlo samples. Different widths are obtained for each $\mathrm{W}^{+} \mathrm{W}^{-} \rightarrow \mathrm{qq} \ell \nu_{\ell}$ decay channel. The shapes of the background distributions and the background fractions are determined from Monte Carlo. The background fractions are held constant in the fit. The fit is restricted to the range $70<m_{\text {rec }}<88 \mathrm{GeV}$.

In the $\mathrm{W}^{+} \mathrm{W}^{-} \rightarrow$ qqqq channel events are divided into four and five jet samples which are fitted separately. The division is made on the basis of the 5-C kinematic fit probability. Events are reconstructed as five jets if any of the ten possible jet-pairings in the five jet assignment gives a fit probability which is greater than twice that of highest probability of the three jet-pairings for the four jet hypothesis. For both four jet and five jet samples, the fitting function $S\left(m_{\text {rec }}\right)$ is multiplied by $G\left(m_{\text {rec }}\right)=\exp \left[-\left(m_{0}-m_{\text {rec }}\right)^{2} / 2 \sigma^{2}\right]$. This empirical choice provides a good description of the reconstructed mass spectra in the fit range for $\mathrm{W}^{+} \mathrm{W}^{-} \rightarrow$ qqqq events. The value of $\sigma$ is determined from Monte Carlo. For the four jet $\mathrm{W}^{+} \mathrm{W}^{-} \rightarrow$ qqqq sample either one or two jet-pairings are used following the procedure described in [9]. In the five jet sample, a jet-pairing likelihood is used. Here the variables used in the likelihood are those used for the reweighting fit described in Section 4.1, with the exception that the 5 -C fit mass is not included. 
In contrast to the procedure employed for the reweighting method, the $\mathrm{W}^{+} \mathrm{W}^{-} \rightarrow \mathrm{qq} \ell \nu_{\ell}$ events are not divided into subsamples according to $\sigma_{\text {rec }}$ nor are the $\mathrm{W}^{+} \mathrm{W}^{-} \rightarrow$ qqqq events divided into subsamples according to the jet-pairing likelihood. However, $\mathrm{W}^{+} \mathrm{W}^{-} \rightarrow \mathrm{qq} \tau \nu_{\tau}$ events are sub-divided into fully-leptonic and semi-leptonic decays of the tau lepton.

The fitted mass, $m_{0}$, must be corrected for offsets not accounted for in the fit, e.g. from initial-state radiation and event selection. The correction is determined using fully simulated Monte Carlo samples generated at different values of $M_{\mathrm{W}}$ with the expected background contributions included and is found to depend linearly on $M_{\mathrm{W}}$. The results from the $\mathrm{W}^{+} \mathrm{W}^{-} \rightarrow$ qqqq and $\mathrm{W}^{+} \mathrm{W}^{-} \rightarrow \mathrm{qq} \ell \nu_{\ell}$ channels, after correction, are given in Table 3. The expected statistical error on the combined $\mathrm{W}$ mass measurement is $78 \pm 2 \mathrm{MeV}$.

\subsubsection{Convolution fit}

The convolution method [12, 16] attempts to exploit all available information by constructing a likelihood curve for each selected event. The likelihood is calculated using a functional form

$$
\mathcal{L}\left(M_{\mathrm{W}}, m_{\mathrm{rec}}\right)=p_{s} \mathcal{P}_{s}\left(M_{\mathrm{W}}, m_{\mathrm{rec}}\right),
$$

where $p_{s}$ is the probability of a candidate event being a true signal event and $\mathcal{P}_{s}$ is the probability function for $M_{\mathrm{W}}$ given the observed reconstructed mass, $m_{\mathrm{rec}}$. The function $\mathcal{P}_{s}\left(M_{\mathrm{W}}, m_{\mathrm{rec}}\right)$ is defined as

$$
\mathcal{P}_{s}\left(M_{\mathrm{W}}, m_{\mathrm{rec}}\right)=\mathrm{BW}\left(M_{\mathrm{W}}, m, s^{\prime}\right) \otimes \operatorname{ISR}\left(s, s^{\prime}\right) \otimes \mathrm{R}\left(m, m_{\mathrm{rec}}\right),
$$

where $\mathrm{BW}\left(M_{\mathrm{W}}, m, s^{\prime}\right)$ is the relativistic Breit-Wigner function for producing off-shell $\mathrm{W}$ bosons with mass $m$ for a $\mathrm{W}$ mass of $M_{\mathrm{W}}$ including the effects of phase space. $\Gamma_{\mathrm{W}}$ is fixed relative to $M_{\mathrm{W}}$ using Equation 1. The radiator function, $\operatorname{ISR}\left(s, s^{\prime}\right)$, is used to account for the effects of ISR (on a statistical basis). The inclusion of this term accounts for the fact that in the kinematic fit the observed energy is constrained to $\sqrt{s}$, where in reality the effective centre-of-mass energy, $\sqrt{s^{\prime}}$, depends on the amount of ISR. Finally, $\mathrm{R}\left(m, m_{\mathrm{rec}}\right)$ is the probability density function relating the experimentally reconstructed mass, $m_{\text {rec }}$, to the average of the two true masses, $m$, of the off-shell $\mathrm{W}$ bosons in the event.

In the $\mathrm{W}^{+} \mathrm{W}^{-} \rightarrow \mathrm{qq} \ell \nu_{\ell}$ channels, the full error information on the fitted mass of each event is used in an unbinned maximum likelihood fit. In a previous OPAL publication [10], the event probability function, $\mathrm{R}\left(m, m_{\mathrm{rec}}\right)$, was assumed to be Gaussian with its central value and standard deviation taken from a 2-C kinematic fit. In this analysis, the 2-C fit is modified, replacing the equal $\mathrm{W}$ mass constraint by one in which the masses are individually constrained to a given $\mathrm{W}$ boson mass, $m$. The $\chi^{2}$ distribution from this fit is converted into the event probability density function, $\mathrm{R}\left(m, m_{\mathrm{rec}}\right)$, which is non-Gaussian.

In the $\mathrm{W}^{+} \mathrm{W}^{-} \rightarrow$ qqqq channel, all events are forced into a five jet topology. A relative likelihood, constructed from the difference between the reconstructed masses in a 4-C kinematic fit, is used to select on average about three jet-pairings per event thereby reducing significantly the combinatorial background. In the Monte Carlo $92 \%$ of selected events the correct jet-pairing combination is used in the fit. The convolution is performed in two dimensions, namely the reconstructed masses of the two $\mathrm{W}$ bosons, with joint probability density functions corresponding to each jet-pairing obtained from the 4-C kinematic fit. The probability density functions for the different combinations are added to form an event probability density function [12].

In both channels the log-likelihood curves from each selected event are summed to yield a single curve from which a fitted mass is determined. The fitted mass is corrected for offsets not accounted for 
in the fit in the manner described for the Breit-Wigner fit. The results from the $\mathrm{W}^{+} \mathrm{W}^{-} \rightarrow \mathrm{qqqq}$ and $\mathrm{W}^{+} \mathrm{W}^{-} \rightarrow \mathrm{qq} \ell \nu_{\ell}$ channels, after all corrections, are given in Table 3. The expected statistical error on the combined $\mathrm{W}$ mass measurement is $72 \pm 2 \mathrm{MeV}$. The total combined expected error, statistical and systematic, from the convolution method is slightly smaller than that obtained from the reweighting fit. However, at present the reweighting fit is the only method in which a simultaneous fit for $\left(M_{\mathrm{W}}, \Gamma_{\mathrm{W}}\right)$ is implemented. For this reason, and for consistency with previous OPAL publications, the reweighting method is retained for the results given in this publication.

\section{Systematic Uncertainties}

The systematic uncertainties for the measurement of $M_{\mathrm{W}}$ using the reweighting method are estimated as described below and summarised in Table 1 . The contributions from each of the sources are added in quadrature to yield the total systematic uncertainty. All contributions were evaluated for both the $M_{\mathrm{W}}$ and $\Gamma_{\mathrm{W}}$ determinations. For the alternative analyses, the systematics are estimated similarly and yield comparable results. The determination of the individual systematic uncertainties is described below.

\section{$5.1 \quad$ Beam energy}

The average LEP beam energy is currently known with a precision of $20 \mathrm{MeV}$ 19 which leads to a systematic uncertainty of $16 \mathrm{MeV}$ on $M_{\mathrm{W}}$ and $2 \mathrm{MeV}$ on $\Gamma_{\mathrm{W}}$. The RMS spread in the LEP centre-ofmass energy of $237 \pm 12 \mathrm{MeV}$ 19 and the average difference between the electron and positron beam energies results in negligible impact on the measurement of $M_{\mathrm{W}}$. The Monte Carlo does not include the intrinsic beam energy spread. This introduces a bias of $+10 \mathrm{MeV}$ to the value of $\Gamma_{\mathrm{W}}$ obtained from the reweighting fit. A $-10 \mathrm{MeV}$ correction is applied and the size of the correction taken as an additional systematic uncertainty on the measurement $\Gamma_{\mathrm{W}}$.

\subsection{Initial state radiation}

The main Koralw Monte Carlo samples include an $\mathcal{O}\left(\alpha^{3}\right)$ treatment of initial state radiation (ISR). The systematic error associated with ISR is estimated by reweighting the generated KorALW events to correspond to an $\mathcal{O}\left(\alpha^{2}\right)$ or $\mathcal{O}(\alpha)$ treatment of ISR using the matrix elements calculated in KoRALW. The resulting shifts from the comparison of $\mathcal{O}\left(\alpha^{3}\right)$ to $\mathcal{O}(\alpha)$ are less than $2 \mathrm{MeV}$ and are neglected.

\section{$5.3 \quad$ Full $\mathcal{O}(\alpha)$ corrections}

In the process $\mathrm{e}^{+} \mathrm{e}^{-} \rightarrow 4 f \gamma$ ISR diagrams dominate. The accuracy of the treatment of ISR in the KORALW generator is sufficient for this analysis. However the KORALW generator does not include all $\mathcal{O}(\alpha)$ effects. For example, interference between ISR and FSR graphs and photon radiation from the $\mathrm{W}$ bosons are not implemented. Recent calculations using the double-pole approximation indicate that possible mass biases of order $10 \mathrm{MeV}$ could arise [29]. However, these estimates were based on generator level studies which compared mass distributions from the full $\mathcal{O}(\alpha)$ treatment with the

corresponding Born level treatment which does not include any final state radiation. To obtain a proper estimate of the potential mass bias when applied to the experimental measurement it would 
be necessary to use the full OPAL detector simulation and mass reconstruction procedure. Currently this is not possible since the necessary Monte Carlo programs are not yet available. For this paper possible systematic biases from the full $\mathcal{O}(\alpha)$ treatment of photon radiation are not included since the size of the effect is significantly smaller than the current statistical precision of the $M_{\mathrm{W}}$ measurement.

\subsection{Hadronisation in $\mathrm{W}^{ \pm} \rightarrow \mathrm{q} \overline{\mathrm{q}}$ decays}

The Koralw Monte Carlo samples taken as references in the extraction of $M_{\mathrm{W}}$ use the Lund string model as implemented in JETSET for the simulation of the hadronisation/ of $\mathrm{W}^{ \pm} \rightarrow \mathrm{q} \overline{\mathrm{q}}$ decays. The JETSET model was tuned to OPAL hadronic data recorded at the $\mathrm{Z}^{0}$ resonance [30]. The model was tuned to describe event shape variables and inclusive particle production rates. To assess a possible systematic error from the uncertainties in the tuning of the JETSET model, the JETSET parameters $\sigma_{q}, b, \Lambda_{\mathrm{QCD}}$ and $Q_{0}$ are each varied in turn by $\pm 1 \sigma$ about their tuned values at the $\mathrm{Z}^{0}$ [30]. The maximum resulting biases to the fitted values of $M_{\mathrm{W}}$ from these high statistics samples are $30 \mathrm{MeV}$ and $20 \mathrm{MeV}$ in the qq $\ell \nu_{\ell}$ and qqqq channels respectively. In addition, an earlier OPAL tune of the JETSET model is used. This version was tuned to describe global event shape variables alone [31]. The resulting differences in the fitted value of $M_{\mathrm{W}}$ using the earlier OPAL tune are $(-33 \pm 14) \mathrm{MeV}$ and $(-30 \pm 12) \mathrm{MeV}$ in the $\mathrm{W}^{+} \mathrm{W}^{-} \rightarrow \mathrm{qqqq}$ and $\mathrm{W}^{+} \mathrm{W}^{-} \rightarrow \mathrm{qq} \ell \nu_{\ell}$ channels respectively.

As a further test of hadronisation uncertainties, the JETSET string model is replaced by the HERWIG cluster model. For this comparison, two samples were generated using the same $\mathrm{W}^{+} \mathrm{W}^{-}$final states and differing only in the hadronisation modelling. The resulting fitted mass values from the reweighting method from Jetset and Herwig are compared. Differences (Jetset-HeRwig) of $(-18 \pm 14) \mathrm{MeV}$ and $(-11 \pm 12) \mathrm{MeV}$ are obtained for the $\mathrm{W}^{+} \mathrm{W}^{-} \rightarrow \mathrm{qq} \ell \nu_{\ell}$ and $\mathrm{W}^{+} \mathrm{W}^{-} \rightarrow$ qqqq channels. A similar comparison is made using the ARIADne colour dipole model [32]. Differences (Jetset-Ariadne) of $(+15 \pm 14) \mathrm{MeV}$ and $(+6 \pm 12) \mathrm{MeV}$ are obtained for the $\mathrm{W}^{+} \mathrm{W}^{-} \rightarrow \mathrm{qq} \ell \nu_{\ell}$ and $\mathrm{W}^{+} \mathrm{W}^{-} \rightarrow \mathrm{qqqq}$ channels.

On the basis of these comparisons systematic uncertainties of $30 \mathrm{MeV}$ are assigned to the $\mathrm{W}$ mass measurements from both $\mathrm{W}^{+} \mathrm{W}^{-} \rightarrow \mathrm{qq} \ell \nu_{\ell}$ and $\mathrm{W}^{+} \mathrm{W}^{-} \rightarrow$ qqqq. The hadronisation uncertainty is taken to be fully correlated between the two channels. For the measurement of $\Gamma_{\mathrm{W}}$ the corresponding systematic errors in the $\mathrm{W}^{+} \mathrm{W}^{-} \rightarrow \mathrm{qq} \ell \nu_{\ell}$ and $\mathrm{W}^{+} \mathrm{W}^{-} \rightarrow$ qqqq channels are $25 \mathrm{MeV}$ and $60 \mathrm{MeV}$ respectively.

\subsection{Detector calibration and simulation}

The effects of detector mis-calibrations and deficiencies in the Monte Carlo are investigated by varying the jet and lepton energy scales over reasonable ranges. The ranges used for the systematic variations are dependent on polar angle and are determined from detailed comparisons of data and Monte Carlo utilising both data recorded at $189 \mathrm{GeV}$ and $3.1 \mathrm{pb}^{-1}$ of data collected at $\sqrt{s} \approx M_{\mathrm{Z}}$ during 1998. From $\mathrm{e}^{+} \mathrm{e}^{-} \rightarrow \mathrm{e}^{+} \mathrm{e}^{-}$events the electro-magnetic calorimeter energy scale is known to $0.3 \%$. Similarly from $\mathrm{e}^{+} \mathrm{e}^{-} \rightarrow \mathrm{Z}^{0} \rightarrow \mu^{+} \mu^{-}$events, the uncertainty on momentum scale from the tracking detectors is determined to be $0.3 \%$. These scale uncertainties dominate the detector related systematics in the $\mathrm{W}^{+} \mathrm{W}^{-} \rightarrow \mathrm{qqe} \nu_{\mathrm{e}}$ and $\mathrm{W}^{+} \mathrm{W}^{-} \rightarrow \mathrm{qq} \mu \nu_{\mu}$ channels, and are $14 \mathrm{MeV}$ and $16 \mathrm{MeV}$ respectively. Uncertainties on energy (momentum) resolution and angular resolution are also evaluated but have much smaller impact. Uncertainties on the jet energy scale are determined from $\mathrm{e}^{+} \mathrm{e}^{-} \rightarrow \mathrm{Z}^{0} \rightarrow \mathrm{q} \overline{\mathrm{q}}$

\footnotetext{
${ }^{2}$ In this paper hadronisation refers to the process whereby the quarks produced in $\mathrm{W}$ decays produce the hadrons observed in the detector including the development of the parton shower.
} 
events to be $0.2 \%-1.0 \%$ depending on the polar angle. The dominant effect of the jet energy scale uncertainty is a $6 \mathrm{MeV}$ uncertainty in the $\mathrm{W}^{+} \mathrm{W}^{-} \rightarrow \mathrm{qq} \tau \nu_{\tau}$ channel. In the $\mathrm{W}^{+} \mathrm{W}^{-} \rightarrow$ qqqq channel the sensitivity to the overall jet energy scale is greatly reduced due to the kinematic fit. Here the dominant effect is from the global uncertainty in the jet polar angle scale, i.e. the relative length-towidth scale of the detector, resulting in a $3 \mathrm{MeV}$ uncertainty on the $\mathrm{W}$ mass. The linearity of the jet energy scale is checked using $\mathrm{e}^{+} \mathrm{e}^{-} \rightarrow \mathrm{Z}^{0} \rightarrow \mathrm{q} \overline{\mathrm{q}}$ events with a clear three-jet topology. For the measurement of $\Gamma_{\mathrm{W}}$ the main systematic error arises from uncertainties in the jet energy scale and resolution and is $47 \mathrm{MeV}$ in both $\mathrm{W}^{+} \mathrm{W}^{-} \rightarrow \mathrm{qq} \ell \nu_{\ell}$ and $\mathrm{W}^{+} \mathrm{W}^{-} \rightarrow$ qqqq channels.

\subsection{Four-fermion modelling}

Possible systematic effects associated with the modelling of the four-fermion final state, including interference between $\mathrm{W}^{+} \mathrm{W}^{-}$diagrams and other four-fermion processes, are investigated by comparing the grc4f and the ExCALIBUR [33] generators. The comparison is performed using the ratio of the ExCALibur to grc4f four-fermion matrix elements to reweight the KoralW Monte Carlo sample. This procedure avoids introducing an additional statistical error associated with generating additional Monte Carlo. The resulting shift in the reconstructed mass is $1 \pm 1 \mathrm{MeV}$ in the $\mathrm{W}^{+} \mathrm{W}^{-} \rightarrow \mathrm{qq} \ell \nu_{\ell}$ channel and $0 \pm 1 \mathrm{MeV}$ in the $\mathrm{W}^{+} \mathrm{W}^{-} \rightarrow$ qqqq channel. Similar results are obtained when the ExcALIBUR Monte Carlo is reweighted using the ratio of the grc4f to ExCALIBUR four-fermion matrix elements. Consequently no additional systematic uncertainty is assigned. The corresponding systematic uncertainties on the measurement of $\Gamma_{\mathrm{W}}$, of order $30 \mathrm{MeV}$, are determined by the statistical precision of the comparisons. In the $\mathrm{W}^{+} \mathrm{W}^{-} \rightarrow \mathrm{qqe} \nu_{\mathrm{e}}$ channel an additional $5 \mathrm{MeV}$ uncertainty is assigned, associated with using the Breit-Wigner functions in the reweighting fit.

\subsection{Background treatment}

Uncertainties in the modelling of background processes mainly arise from the simulation of the $\mathrm{Z}^{0} / \gamma \rightarrow \mathrm{q} \overline{\mathrm{q}}$ background in the $\mathrm{W}^{+} \mathrm{W}^{-} \rightarrow \mathrm{qq} \tau \nu_{\tau}$ and $\mathrm{W}^{+} \mathrm{W}^{-} \rightarrow$ qqqq channels. A number of systematic checks of the normalisation and shape of the $\mathrm{Z}^{0} / \gamma \rightarrow \mathrm{q} \overline{\mathrm{q}}$ background are performed. The background normalisation in the reweighting fit is varied by one standard deviation, using the uncertainties evaluated in [25]. The Herwig cluster model is used as an alternative to the LuND string model used in PүтніA. In addition, for the $\mathrm{W}^{+} \mathrm{W}^{-} \rightarrow$ qqqq channel, data taken at $\sqrt{s} \approx M_{\mathrm{Z}}$, scaled by $\left(189 \mathrm{GeV} / M_{\mathrm{Z}}\right)$, are also substituted for the $\mathrm{Z}^{0} / \gamma \rightarrow \mathrm{q} \overline{\mathrm{q}}$ background. The sum in quadrature of the above systematic shifts leads to the assignment of a background systematic error of $6 \mathrm{MeV}$ in the $\mathrm{W}^{+} \mathrm{W}^{-} \rightarrow$ qqqq channel and $8 \mathrm{MeV}$ in the $\mathrm{W}^{+} \mathrm{W}^{-} \rightarrow \mathrm{qq} \ell \nu_{\ell}$ channel. The systematic error on $\Gamma_{\mathrm{W}}$ due to uncertainties in background modelling is $25 \mathrm{MeV}$ and $41 \mathrm{MeV}$ in the $\mathrm{W}^{+} \mathrm{W}^{-} \rightarrow \mathrm{qq} \ell \nu_{\ell}$ and $\mathrm{W}^{+} \mathrm{W}^{-} \rightarrow$ qqqq channels respectively arising from differences between JeTSET and HeRwIG and uncertainties on the accepted background cross section.

\subsection{Monte Carlo statistics}

The finite statistics of the Monte Carlo samples used in the reweighting procedure result in an additional uncertainty of $10 \mathrm{MeV}$ on the $\mathrm{W}$ mass determined separately in the $\mathrm{W}^{+} \mathrm{W}^{-} \rightarrow$ qqqq and $\mathrm{W}^{+} \mathrm{W}^{-} \rightarrow \mathrm{qq} \ell \nu_{\ell}$ channels. The corresponding systematic uncertainties for the $\mathrm{W}$ width measurement are $51 \mathrm{MeV}$. 


\subsection{Final state interactions (FSI)}

A significant bias to the apparent $\mathrm{W}$ mass measured in the $\mathrm{W}^{+} \mathrm{W}^{-} \rightarrow$ qqqq channel could arise if the hadronisation of the two $\mathrm{W}$ bosons is not independent [16, 34]. At LEP2 energies, the decay length for $\mathrm{W}$ bosons is about $0.1 \mathrm{fm}$, significantly less than the typical hadronisation scale, approximately $1 \mathrm{fm}$. The hadronisation of the two $\mathrm{W}$ bosons in the $\mathrm{W}^{+} \mathrm{W}^{-} \rightarrow$ qqqq channel may have space-time overlap allowing possible final state interactions between the decay products of different $\mathrm{W}$ bosons. Two possible sources of final state interactions, both leading to non-independent hadronisation, have been widely considered: Colour Reconnection (CR) and Bose-Einstein Correlations (BEC). Whether these effects play a significant role at LEP2 has yet to be experimentally determined. As a result, to assign a systematic error related to possible final state interactions it is necessary to rely on phenomenological models. The strategy adopted here is to consider only those models for CR and BEC which are consistent with both LEP1 and LEP2 data.

The CR systematic error refers to possible biases in the $\mathrm{W}$ mass measurement arising from QCD reconnection effects in the non-perturbative phase. Reconnection effects in the perturbative phase, i.e. multiple gluon exchange between quarks from different $\mathrm{W}$ bosons, have been shown to be small [35]. To investigate the systematic biases originating from non-perturbative CR effects, the models implemented in the Pythia and Ariadne [32] Monte Carlo generators are studied. In both cases the results from the mass fit to a Monte Carlo sample including CR effects are compared to the results obtained from the corresponding sample without colour reconnection. The results are summarised in Table 5. The largest biases are seen in the ArIAdne 2 and Ariadne 3 models 5 . However, these models have been strongly disfavoured by studies of three jet events in LEP1 $\mathrm{Z}^{0}$ data 37. For this reason the current ARIADNE implementation of CR is not used to assign a systematic uncertainty. Of the remaining models considered, the SK I model [35] produces the largest bias. The SK Monte Carlo samples use the JETSET tune of [30], where the parton shower cut-off, which directly influences the behaviour of the CR model [35], was set to $1.9 \mathrm{GeV}$. The strength parameter $k_{i}$ (also called $\rho$ ) of the SK I model was set to 0.9, corresponding to $35 \%$ of events being reconnected. A systematic uncertainty of $66 \mathrm{MeV}$ on $M_{\mathrm{W}}$ is assigned. The corresponding uncertainty on $\Gamma_{\mathrm{W}}$ is $68 \mathrm{MeV}$.

As is the case in $\mathrm{Z}^{0}$ decays, BEC between like-sign charged pions are observed in $\mathrm{W}^{ \pm}$decays at LEP2 [38]. These correlations are not implemented in the standard Monte Carlo programs used in this analysis. Correlations between pions from the same $\mathrm{W}$ do not affect significantly the global jet properties and are unlikely to bias the $M_{\mathrm{W}}$ measurement. If BEC exist between pions from different $\mathrm{W}$ bosons in $\mathrm{W}^{+} \mathrm{W}^{-} \rightarrow$ qqqq events, significant biases to the reconstructed value of $M_{\mathrm{W}}$ may arise. To investigate possible systematic biases from BEC, a sample of $\mathrm{W}^{+} \mathrm{W}^{-} \rightarrow$ qqqq events is produced using the Pythia Monte Carlo generator which includes BEC as described in [39]. For the evaluation of the systematic error the BEC model $B E_{32}$ of 39 with $\lambda=1.0$ and $R=0.42 \mathrm{fm}$ is used. The difference in the fitted $\mathrm{W}$ mass between a sample generated with BEC between different $\mathrm{W}$ bosons and a sample generated with BEC only between particles from the same $\mathrm{W}$ is $+67 \pm 14 \mathrm{MeV}$ (different-same). The resulting systematic uncertainties on $M_{\mathrm{W}}$ and $\Gamma_{\mathrm{W}}$ from $\mathrm{BEC}$ in the $\mathrm{W}^{+} \mathrm{W}^{-} \rightarrow$ qqqq channel are $67 \mathrm{MeV}$ and $39 \mathrm{MeV}$ respectively.

\subsection{Fit procedure}

A comparison of the results from the reweighting method with those from the Breit-Wigner and convolution fits, summarised in Table 3, is used to test for residual biases in the reweighting method. The

\footnotetext{
${ }^{3}$ Following the notation used in $[36,37]$.
} 
expected RMS of the differences of the results from the three methods are determined using an ensemble of Monte Carlo subsamples including background, each corresponding to an integrated luminosity of $183 \mathrm{pb}^{-1}$. For each subsample the difference in the fitted $M_{\mathrm{W}}$ determined using the reweighting method and that determined using each of the other methods is calculated for the $\mathrm{W}^{+} \mathrm{W}^{-} \rightarrow$ qqqq and $\mathrm{W}^{+} \mathrm{W}^{-} \rightarrow \mathrm{qq} \ell \nu_{\ell}$ channels. These differences have mean values consistent with zero and RMS values of approximately $54 \mathrm{MeV}$ and $41 \mathrm{MeV}(62 \mathrm{MeV}$ and $84 \mathrm{MeV}$ ) when comparing the reweighting fits to the Breit-Wigner (convolution) fits in the $\mathrm{W}^{+} \mathrm{W}^{-} \rightarrow \mathrm{qq} \ell \nu_{\ell}$ and $\mathrm{W}^{+} \mathrm{W}^{-} \rightarrow$ qqqq samples, respectively. Since the alternative analyses yield results consistent with those obtained using the default reweighting analysis, no additional systematic error is assigned.

\section{Results}

For the reweighting method described in Section 4.2, the results of a simultaneous fit to $M_{\mathrm{W}}$ and $\Gamma_{\mathrm{W}}$ from the combined $\mathrm{W}^{+} \mathrm{W}^{-} \rightarrow$ qqqq and $\mathrm{W}^{+} \mathrm{W}^{-} \rightarrow \mathrm{qq} \ell \nu_{\ell}$ event samples are

$$
\begin{aligned}
M_{\mathrm{W}} & =80.451 \pm 0.076 \text { (stat.) } \pm 0.050 \text { (syst.) } \mathrm{GeV}, \\
\Gamma_{\mathrm{W}} & =2.09 \pm 0.18 \text { (stat.) } \pm 0.09 \text { (syst.) GeV. }
\end{aligned}
$$

The correlation coefficient between $M_{\mathrm{W}}$ and $\Gamma_{\mathrm{W}}$ is 0.04 . For this fit, the central values are determined by adding the log-likelihood curves from the $\mathrm{W}^{+} \mathrm{W}^{-} \rightarrow \mathrm{qqqq}$ and $\mathrm{W}^{+} \mathrm{W}^{-} \rightarrow \mathrm{qq} \ell \nu_{\ell}$ channels including the effects of systematic uncertainties. The likelihood contours for this fit are displayed in Figure 1 .

A one parameter fit for the mass is performed by constraining the width using Equation 11 to give $M_{\mathrm{W}}=80.402 \pm 0.104$ (stat.) \pm 0.101 (syst.) $\mathrm{GeV}$ in the $\mathrm{W}^{+} \mathrm{W}^{-} \rightarrow$ qqqq channel, and $M_{\mathrm{W}}=$ $80.478 \pm 0.104$ (stat.) \pm 0.038 (syst.) $\mathrm{GeV}$ in the $\mathrm{W}^{+} \mathrm{W}^{-} \rightarrow \mathrm{qq} \ell \nu_{\ell}$ channel. The combined result, taking into account the correlated systematics between the $\mathrm{W}^{+} \mathrm{W}^{-} \rightarrow \mathrm{qqqq}$ and $\mathrm{W}^{+} \mathrm{W}^{-} \rightarrow \mathrm{qq} \ell \nu_{\ell}$ channels, is

$$
M_{\mathrm{W}}=80.451 \pm 0.076 \text { (stat.) } \pm 0.049 \text { (syst.) GeV. }
$$

Due to the larger systematic errors in the $\mathrm{W}^{+} \mathrm{W}^{-} \rightarrow$ qqqq channel, mainly from FSI, it carries a reduced weight of 0.36 in the combination. The combined $\mathrm{W}^{+} \mathrm{W}^{-} \rightarrow \mathrm{qqqq}$ and $\mathrm{W}^{+} \mathrm{W}^{-} \rightarrow \mathrm{qq} \ell \nu_{\ell}$ results from the alternative analyses, after all corrections, are for the Breit-Wigner fit, $M_{\mathrm{W}}=$ $80.436 \pm 0.078$ (stat.) \pm 0.050 (syst.) $\mathrm{GeV}$, and for the convolution fit, $M_{\mathrm{W}}=80.378 \pm 0.073$ (stat.) \pm 0.051(syst.) GeV.

\subsection{Combination with previous data}

The results presented in this paper, based on $183 \mathrm{pb}^{-1}$ of data, are combined with previous OPAL measurements of $M_{\mathrm{W}}$ from direct reconstruction at $\sqrt{s} \approx 172 \mathrm{GeV}[9]$ and at $\sqrt{s} \approx 183 \mathrm{GeV}$ [10]. The results in the $\mathrm{W}^{+} \mathrm{W}^{-} \rightarrow \mathrm{qq} \ell \nu_{\ell}$ and $\mathrm{W}^{+} \mathrm{W}^{-} \rightarrow$ qqqq channels are combined independently taking into account systematic uncertainties which are correlated between data from the different centre-of-mass energies, giving:

$$
\begin{aligned}
M_{\mathrm{W}}\left(\mathrm{W}^{+} \mathrm{W}^{-} \rightarrow \mathrm{qq} \ell \nu_{\ell}\right) & =80.441 \pm 0.086 \text { (stat.) } \pm 0.034 \text { (syst.) } \pm 0.017 \text { (lep) } \mathrm{GeV} \\
M_{\mathrm{W}}\left(\mathrm{W}^{+} \mathrm{W}^{-} \rightarrow \mathrm{qqqq}\right) & =80.409 \pm 0.093 \text { (stat.) } \pm 0.034 \text { (syst.) } \pm 0.016 \text { (lep) } \pm 0.094 \text { (fsi) GeV }
\end{aligned}
$$

where the uncertainties from the LEP beam energy (lep) and from final state interactions (fsi) are quoted separately. In the combination the systematic uncertainties for the colour reconnection and Bose-Einstein correlations of the previous data were set to those presented in this paper. 
The difference between the fitted $M_{\mathrm{W}}$ in the $\mathrm{W}^{+} \mathrm{W}^{-} \rightarrow$ qqqq and $\mathrm{W}^{+} \mathrm{W}^{-} \rightarrow \mathrm{qq} \ell \nu_{\ell}$ channels is $\Delta M_{\mathrm{W}} \equiv\left(M_{\mathrm{W}}^{\mathrm{qqqq}}-M_{\mathrm{W}}^{\mathrm{qq} \ell \nu_{\ell}}\right)=-0.032 \pm 0.127$ (stat.) \pm 0.047 (syst.) $\mathrm{GeV}$. A significant non-zero value for $\Delta M_{\mathrm{W}}$ could indicate that FSI effects are biasing the value of $M_{\mathrm{W}}$ determined from $\mathrm{W}^{+} \mathrm{W}^{-} \rightarrow$ qqqq events. The systematic error on the quoted value of $\Delta M_{\mathrm{W}}$ does not include contributions from $\mathrm{CR} / \mathrm{BEC}$ effects. For the $\Delta M_{\mathrm{W}}$ measurement the hadronization systematic uncertainties in the $\mathrm{W}^{+} \mathrm{W}^{-} \rightarrow$ qqqq and $\mathrm{W}^{+} \mathrm{W}^{-} \rightarrow \mathrm{qq} \ell \nu_{\ell}$ channels are taken to be uncorrelated; a conservative choice in the absence of a good estimate of the true correlation coefficient.

The $M_{\mathrm{W}}$ results from direct reconstruction in the $\mathrm{W}^{+} \mathrm{W}^{-} \rightarrow \mathrm{qq} \ell \nu_{\ell}$ and $\mathrm{W}^{+} \mathrm{W}^{-} \rightarrow$ qqqq channels are combined with the OPAL measurement of $M_{\mathrm{W}}$ from the $\mathrm{W}^{+} \mathrm{W}^{-}$production cross-section at $\sqrt{s} \approx 161 \mathrm{GeV}[8]$. The combination is made assuming that the mass measurements from direct reconstruction and from the threshold cross-section are uncorrelated, apart from the uncertainty associated with the LEP beam energy, which is taken to be fully correlated. The direct reconstruction measurements from the $\mathrm{W}^{+} \mathrm{W}^{-} \rightarrow \mathrm{qq} \ell \nu_{\ell}$ and $\mathrm{W}^{+} \mathrm{W}^{-} \rightarrow$ qqqq channels from three different centre-of-mass energies are combined using the full covariance matrix. The combined result is

$$
M_{\mathrm{W}}=80.432 \pm 0.066 \text { (stat.) } \pm 0.032 \text { (syst.) } \pm 0.028 \text { (fsi) } \pm 0.017 \text { (lep) GeV. }
$$

The measurement of $\Gamma_{\mathrm{W}}$ presented in this paper is combined with the previous OPAL measurements from direct reconstruction [9, 10] to give

$$
\Gamma_{\mathrm{W}}=2.04 \pm 0.16 \text { (stat.) } \pm 0.09 \text { (syst.) GeV. }
$$

For the earlier data, with relatively low statistics, the measured statistical error is correlated with the measured width. Consequently, to avoid biasing the combination, the separate width measurements are weighted using the expected statistical error rather than the measured statistical error.

\section{Summary}

Using the $183 \mathrm{pb}^{-1}$ of data recorded by the OPAL detector at a mean centre-of-mass energy of approximately $189 \mathrm{GeV}$, a total of $2088 \mathrm{~W}^{+} \mathrm{W}^{-} \rightarrow \mathrm{qqqq}$ and $\mathrm{W}^{+} \mathrm{W}^{-} \rightarrow \mathrm{qq} \ell \nu_{\ell}$ candidate events are used in a fit constraining $\Gamma_{\mathrm{W}}$ using Equation 11 to obtain a direct measurement of the $\mathrm{W}$ boson mass, $M_{\mathrm{W}}=80.451 \pm 0.076$ (stat.) \pm 0.049 (syst.) $\mathrm{GeV}$, while a second fit is used to determine directly the width of the $\mathrm{W}$ boson, $\Gamma_{\mathrm{W}}=2.09 \pm 0.18$ (stat.) \pm 0.09 (syst.) $\mathrm{GeV}$.

The results described in this paper are combined with the previous OPAL results from data recorded at $\sqrt{s} \approx 161-183 \mathrm{GeV}$ [10]. From this combined data sample the $\mathrm{W}$ boson mass is determined to be

$$
M_{\mathrm{W}}=80.432 \pm 0.066 \text { (stat.) } \pm 0.045 \text { (syst.) GeV. }
$$

This result is consistent with both the other direct measurements and the indirect value inferred from fits to electroweak data [3]. It should be noted that the systematic error is dominated by the uncertainties arising from hadronisation and final state interactions. For future LEP combinations these uncertainties will need to be reduced to benefit from the ultimate LEP combined statistical uncertainty of about $25 \mathrm{MeV}$.

The result for the $\mathrm{W}$ boson width is combined with the previous OPAL result from data recorded at $\sqrt{s} \approx 172-183 \mathrm{GeV}$ to obtain

$$
\Gamma_{\mathrm{W}}=2.04 \pm 0.16 \text { (stat.) } \pm 0.09 \text { (syst.) GeV. }
$$


This measurement of $\Gamma_{\mathrm{W}}$ is in good agreement with the Standard Model expectation and with other direct measurements at LEP [12,13,40] and at the Tevatron 411.

\section{Acknowledgements}

We particularly wish to thank the SL Division for the efficient operation of the LEP accelerator at all energies and for their continuing close cooperation with our experimental group. We thank our colleagues from CEA, DAPNIA/SPP, CE-Saclay for their efforts over the years on the time-of-flight and trigger systems which we continue to use. In addition to the support staff at our own institutions we are pleased to acknowledge the

Department of Energy, USA, National Science Foundation, USA, Particle Physics and Astronomy Research Council, UK, Natural Sciences and Engineering Research Council, Canada, Israel Science Foundation, administered by the Israel Academy of Science and Humanities, Minerva Gesellschaft, Benoziyo Center for High Energy Physics,

Japanese Ministry of Education, Science and Culture (the Monbusho) and a grant under the Monbusho International Science Research Program, Japanese Society for the Promotion of Science (JSPS), German Israeli Bi-national Science Foundation (GIF), Bundesministerium für Bildung und Forschung, Germany, National Research Council of Canada, Research Corporation, USA, Hungarian Foundation for Scientific Research, OTKA T-029328, T023793 and OTKA F-023259. 


\section{References}

[1] S.L. Glashow, J. Iliopoulos, and L. Maiani, Phys. Rev. D2 (1970) 1285;

S. Weinberg, Phys. Rev. Lett. 19 (1967) 1264;

A. Salam, Elementary Particle Theory, ed. N. Svartholm (Almquist and Wiksells, Stockholm, 1968), 367.

[2] ALEPH Collaboration, R. Barate et al., Eur. Phys. J. C14 (2000) 1;

DELPHI Collaboration, "Cross-Sections and Leptonic Forward-Backward Asymmetries from the $\mathrm{Z}^{0}$ running of LEP",CERN-EP/2000-037, Accepted by Eur. Phys. J. C;

L3 Collaboration, M. Acciarri et al., CERN-EP/2000-022, "Measurements of Cross-Sections and Forward Backward Asymmetries at the $\mathrm{Z}^{0}$ Resonance and Determination of Electroweak Parameters" Accepted by Eur. Phys. J. C;

OPAL Collaboration, R. Akers et al., Z. Phys. C61 (1994) 19.

[3] LEP Electroweak Working Group, CERN-EP/2000-016.

[4] ALEPH Collaboration, R. Barate et al., Phys. Lett. B462 (1999) 389 ;

DELPHI Collaboration, P. Abreu et al., Phys. Lett. B459 (1999) 382 ;

L3 Collaboration, M. Acciarri et al., Phys. Lett. B467 (1999) 171 ;

OPAL Collaboration, G. Abbiendi et al., Eur. Phys. J. C8 (1999) 191.

[5] CDF Collaboration, F. Abe et al., Phys. Rev. Lett. 80 (1998) 2767;

CDF Collaboration, F. Abe et al., Phys. Rev. Lett. 80 (1998) 2779;

D0 Collaboration, B. Abbott et al., Phys. Rev. D60 (1999) 052001;

D0 Collaboration, B. Abbott et al., Phys. Rev. D58 (1998) 052001.

[6] G. Degrassi et al., Phys. Lett. B418 (1998) 209.

[7] W.J. Marciano and A. Sirlin, Phys. Rev. D29 (1984) 945.

[8] OPAL Collaboration, K. Ackerstaff et al., Phys. Lett. B389 (1996) 416.

[9] OPAL Collaboration, K. Ackerstaff et al., Eur. Phys. J. C1 (1998) 395.

[10] OPAL Collaboration, G. Abbiendi et al., Phys. Lett. B453 (1999) 138.

[11] ALEPH Collaboration, R. Barate et al., Phys. Lett. B453 (1999) 121.

[12] DELPHI Collaboration, P. Abreu et al., Phys. Lett. B462 (1999) 410.

[13] L3 Collaboration, M. Acciarri et al., Phys. Lett. B454 (1999) 386.

[14] CDF Collaboration, F. Abe et al., Phys. Rev. Lett. 65 (1990) 2243;

CDF Collaboration, F. Abe et al., Phys. Rev. Lett. 75 (1995) 11;

D0 Collaboration, S. Abachi et al., Phys. Rev. Lett. 84 (2000) 222;

UA1 Collaboration, C. Albajar et al., Z. Phys. C44 (1989) 15;

UA2 Collaboration, J. Alitti et al., Phys. Lett. B276 (1992) 354.

[15] C. Caso et al., Eur. Phys. J. C15 (2000) 1.

[16] Physics at LEP2, eds. G. Altarelli, T. Sjöstrand and F. Zwirner, CERN 96-01, (1996).

[17] OPAL Collaboration, K. Ahmet et al., Nucl. Instr. Meth. A305 (1991) 275;

B.E. Anderson et al., IEEE Transactions on Nuclear Science, 41 (1994) 845;

S. Anderson et al., Nucl. Instr. Meth. A403 (1998) 326. 
[18] OPAL Collaboration, G. Abbiendi et al., Eur. Phys. J. C13 (2000) 553.

[19] LEP Energy Working Group, Report 99-01, "Evaluation of the LEP centre-of-mass energy for data taken in 1998", private communication;

LEP Energy Working Group, A. Blondel et al., Eur. Phys. J. C11 (1999) 573.

[20] J. Allison et al., Nucl. Instr. Meth. A317 (1992) 47.

[21] S. Jadach, W. Placzek, M. Skrzypek, B.F.L. Ward and Z. Was, Comp. Phys. Comm. 119 (1999) 272.

[22] J. Fujimoto et al., Comp. Phys. Comm. 100 (1997) 128.

[23] T. Sjöstrand, Comp. Phys. Comm. 82 (1994) 74. Pythia version 6.125 was used for the analysis presented in this paper.

[24] G. Marchesini et al., Comp. Phys. Comm. 67 (1992) 465.

[25] OPAL Collaboration, G. Abbiendi et al., "W $\mathrm{W}^{+} \mathrm{W}^{-}$Production Cross Section and W Branching Fractions in $\mathrm{e}^{+} \mathrm{e}^{-}$Collisions at 189 GeV", CERN-EP/2000-101, submitted to Phys. Lett. B.

[26] N. Brown and W.J. Stirling, Phys. Lett. B252 (1990) 657;

S. Catani et al., Phys. Lett. B269 (1991) 432;

S. Bethke, Z. Kunszt, D. Soper and W.J. Stirling, Nucl. Phys. B370 (1992) 310;

N. Brown and W.J. Stirling, Z. Phys. C53 (1992) 629.

[27] OPAL Collaboration, K. Ackerstaff et al., Eur. Phys. J. C2 (1998) 213;

OPAL Collaboration, G. Abbiendi et al., Eur. Phys. J. C12 (2000) 567.

[28] A. G. Frodesen, O. Skjeggestad, and H. Tofte, "Probability and Statistics in Particle Physics", Universitetsforlaget (1979).

[29] A. Denner, S. Dittmaier, M. Roth and D. Wackeroth, Phys. Lett. B475 (2000) 127.

[30] OPAL Collaboration, G. Alexander et al., Z. Phys. C69 (1996) 543.

[31] OPAL Collaboration, P.D. Acton et al., Z. Phys. C58 (1993) 387.

[32] L. Lönnblad, Z. Phys. C70 (1996) 107.

[33] F.A. Berends, R. Pittau and R. Kleiss, Comp. Phys. Comm. 85 (1995) 437.

[34] A. Ballestrero et al., J. Phys. G24 (1998) 365.

[35] T. Sjöstrand and V.A. Khoze, Phys. Rev. Lett. 72 (1994) 28.

[36] OPAL Collaboration, G. Abbiendi et al., Phys. Lett. B543 (1999) 153.

[37] OPAL Collaboration, G. Abbiendi et al., Eur. Phys. J. C11 (1999) 217.

[38] ALEPH Collaboration, R. Barate et al., Phys. Lett. B478 (2000) 50;

OPAL Collaboration, G. Abbiendi et al., Eur. Phys. J. C8 (1999) 559.

[39] L. Lönnblad and T. Sjöstrand, Eur. Phys. J. C2 (1998) 165.

[40] ALEPH Collaboration, R. Barate et al., CERN-EP/2000-045, " Measurement of the W Mass and Width in $\mathrm{e}^{+} \mathrm{e}^{-}$Collisions at 189 GeV", Submitted to Eur. Phys. J. C.

[41] CDF Collaboration, T. Affolder et al., FERMILAB-PUB-00-085-E, April 2000, "Direct Measurement of the $W$ Boson Width in $\mathrm{p} \overline{\mathrm{p}}$ Collisions at $\sqrt{s}=1.8 \mathrm{TeV}$ ", Submitted to Phys. Rev. Lett. 


\begin{tabular}{|l|ccc|}
\hline Channel & Observed & Expected & Purity \\
\hline $\mathrm{W}^{+} \mathrm{W}^{-} \rightarrow$ qqqq (4-jet) & 701 & 694 & $83 \%$ \\
$\mathrm{~W}^{+} \mathrm{W}^{-} \rightarrow$ qqqq (5-jet) & 269 & 277 & $91 \%$ \\
$\mathrm{~W}^{+} \mathrm{W}^{-} \rightarrow \mathrm{q} \overline{\mathrm{q}} \nu_{\mathrm{e}}$ & 350 & 376 & $98 \%$ \\
$\mathrm{~W}^{+} \mathrm{W}^{-} \rightarrow \mathrm{q} \overline{\mathrm{q}} \mu \nu_{\mu}$ & 365 & 373 & $99 \%$ \\
$\mathrm{~W}^{+} \mathrm{W}^{-} \rightarrow \mathrm{q} \overline{\mathrm{q}} \tau \nu_{\tau}$ & 403 & 403 & $89 \%$ \\
\hline Combined & 2088 & 2123 & $91 \%$ \\
\hline
\end{tabular}

Table 1: Numbers of events used in the W mass and width determination for each channel and all channels combined. Only events surviving the cuts described in Section 4.1 are included. The $\mathrm{W}^{+} \mathrm{W}^{-} \rightarrow \mathrm{q} \overline{\mathrm{q}} \tau \nu_{\tau}$ numbers include some events initially selected as either $\mathrm{W}^{+} \mathrm{W}^{-} \rightarrow \mathrm{qqe} \nu_{\mathrm{e}}$ or $\mathrm{W}^{+} \mathrm{W}^{-} \rightarrow \mathrm{qq} \mu \nu_{\mu}$.

\begin{tabular}{|l|cc|}
\hline Channel & Measured $M_{\mathrm{W}} / \mathrm{GeV}$ & Expected error $/ \mathrm{GeV}$ \\
\hline $\mathrm{W}^{+} \mathrm{W}^{-} \rightarrow \mathrm{qqe} \nu_{\mathrm{e}}$ & $80.375 \pm 0.175$ & 0.164 \\
$\mathrm{~W}^{+} \mathrm{W}^{-} \rightarrow \mathrm{qq} \mu \nu_{\mu}$ & $80.513 \pm 0.163$ & 0.168 \\
$\mathrm{~W}^{+} \mathrm{W}^{-} \rightarrow \mathrm{qq} \tau \nu_{\tau}$ & $80.594 \pm 0.227$ & 0.220 \\
$\mathrm{~W}^{+} \mathrm{W}^{-} \rightarrow \mathrm{qqqq}(4$-jet) & $80.424 \pm 0.114$ & 0.112 \\
$\mathrm{~W}^{+} \mathrm{W}^{-} \rightarrow \mathrm{qqqq}(5$-jet $)$ & $80.290 \pm 0.257$ & 0.230 \\
\hline $\mathrm{W}^{+} \mathrm{W}^{-} \rightarrow \mathrm{qq} \ell \nu_{\ell}$ & $80.478 \pm 0.104$ & 0.104 \\
$\mathrm{~W}^{+} \mathrm{W}^{-} \rightarrow \mathrm{qqqq}$ & $80.402 \pm 0.104$ & 0.100 \\
\hline
\end{tabular}

Table 2: Results using the reweighting method for the fit from $183 \mathrm{pb}^{-1}$ of data taken at $\sqrt{s} \approx$ $189 \mathrm{GeV}$ for each of the channels separately and for the combined $\mathrm{W}^{+} \mathrm{W}^{-} \rightarrow \mathrm{qq} \ell \nu_{\ell}$ channel. Equation [1 is used to constrain $\Gamma_{\mathrm{W}}$ to $M_{\mathrm{W}}$. The expected errors are estimated using an ensemble of Monte Carlo subsamples. The errors obtained in the data are consistent with the RMS spread of the errors obtained from the Monte Carlo subsamples.

\begin{tabular}{|l|c|c|c|}
\hline Channel & $\begin{array}{c}\text { Reweighting } \\
\text { Measured } M_{\mathrm{W}} / \mathrm{GeV}\end{array}$ & $\begin{array}{c}\text { Breit-Wigner fit } \\
\text { Measured } M_{\mathrm{W}} / \mathrm{GeV}\end{array}$ & $\begin{array}{c}\text { Convolution fit } \\
\text { Measured } M_{\mathrm{W}} / \mathrm{GeV}\end{array}$ \\
\hline $\mathrm{W}^{+} \mathrm{W}^{-} \rightarrow \mathrm{qq} \ell \nu_{\ell}$ & $80.48 \pm 0.10 \pm 0.04$ & $80.47 \pm 0.11 \pm 0.04$ & $80.38 \pm 0.11 \pm 0.04$ \\
$\mathrm{~W}^{+} \mathrm{W}^{-} \rightarrow \mathrm{qqqq}$ & $80.40 \pm 0.10 \pm 0.10$ & $80.38 \pm 0.11 \pm 0.10$ & $80.37 \pm 0.09 \pm 0.09$ \\
\hline
\end{tabular}

Table 3: Comparison of $M_{\mathrm{W}}$ fit results obtained using the three different fitting techniques for the $\mathrm{W}^{+} \mathrm{W}^{-} \rightarrow \mathrm{qq} \ell \nu_{\ell}$ and $\mathrm{W}^{+} \mathrm{W}^{-} \rightarrow$ qqqq channels separately. In each case the first uncertainty is statistical and the second systematic. The observed differences are compatible with the expected RMS differences between the results from the different fits. 


\begin{tabular}{|l||c|c||c|c|}
\hline \multicolumn{1}{|c||}{\begin{tabular}{c} 
Systematic errors \\
\multicolumn{1}{c||}{$(\mathrm{MeV})$}
\end{tabular}} & \multicolumn{2}{c||}{$M_{\mathrm{W}}$} & \multicolumn{2}{c|}{$\Gamma_{\mathrm{W}}$} \\
Beam Energy & 16 & 16 & 10 & 10 \\
Hadronisation & 30 & 30 & 60 & 25 \\
Jet energy/Resolution & 4 & 5 & 47 & 47 \\
Lepton energy/Resolution & - & 9 & - & 9 \\
Four Fermion & - & - & 33 & 29 \\
Background & 6 & 8 & 41 & 25 \\
MC statistics & 10 & 10 & 51 & 51 \\
\hline Sub-total & 36 & 38 & 106 & 84 \\
\hline Colour Reconnection & 66 & - & 68 & - \\
Bose-Einstein Correlations & 67 & - & 39 & - \\
\hline Total systematic error & 101 & 38 & 132 & 84 \\
\hline
\end{tabular}

Table 4: Summary of the systematic uncertainties for the fit results. For the fits to determine $M_{\mathrm{W}}$, $\Gamma_{\mathrm{W}}$ is constrained to its Standard Model relation. The uncertainties are given separately for fits to the $\mathrm{W}^{+} \mathrm{W}^{-} \rightarrow$ qqqq and $\mathrm{W}^{+} \mathrm{W}^{-} \rightarrow \mathrm{qq} \ell \nu_{\ell}$ samples. Uncertainties from ISR give negligible contributions to both $M_{\mathrm{W}}$ and $\Gamma_{\mathrm{W}}$. The uncertainty related to the Monte Carlo models of the four-fermion process give is negligible for the measurement of $M_{\mathrm{W}}$.

\begin{tabular}{|c|c|c|}
\hline CR Model & Mass shift (MeV) & Width shift $(\mathrm{MeV})$ \\
\hline SK I & $+66 \pm 8$ & $+68 \pm 22$ \\
SK II & $+3 \pm 8$ & $+20 \pm 22$ \\
SK II' & $+10 \pm 8$ & $+53 \pm 21$ \\
AR 2 & $+85 \pm 8$ & $+128 \pm 22$ \\
AR 3 & $+140 \pm 10$ & $+309 \pm 21$ \\
\hline
\end{tabular}

Table 5: The predicted shifts in the fitted mass and width from the $\mathrm{W}^{+} \mathrm{W}^{-} \rightarrow$ qqqq channel for various colour-reconnection (CR) models. The numbers correspond to the fitted mass (width) using the reweighting method in the reconnected sample minus the fitted mass (width) for the corresponding sample without CR. The errors are statistical only. 


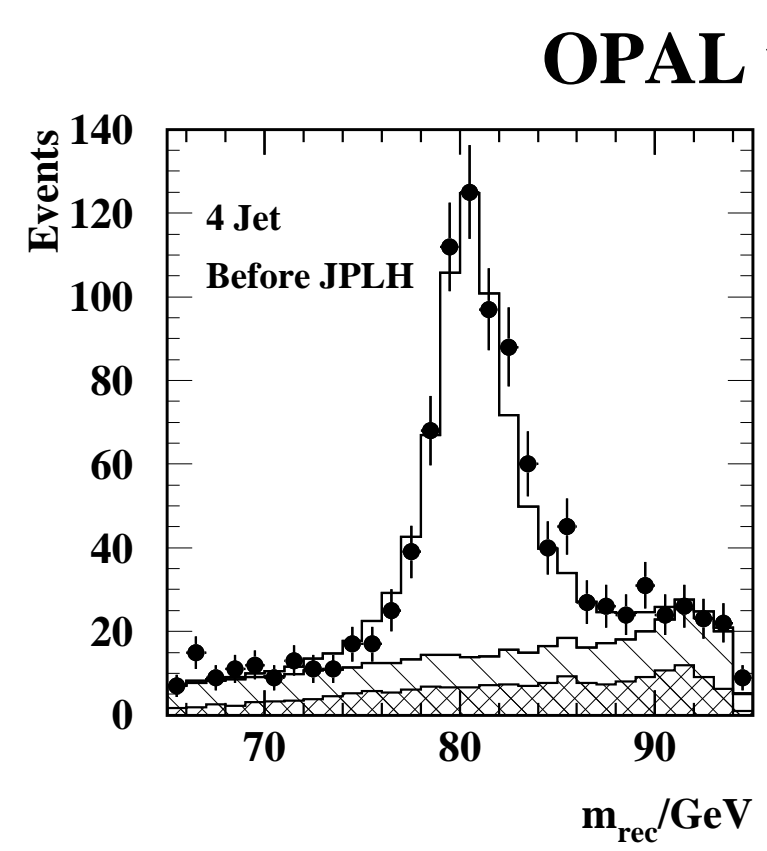

$V_{s}=189 \mathrm{GeV}$
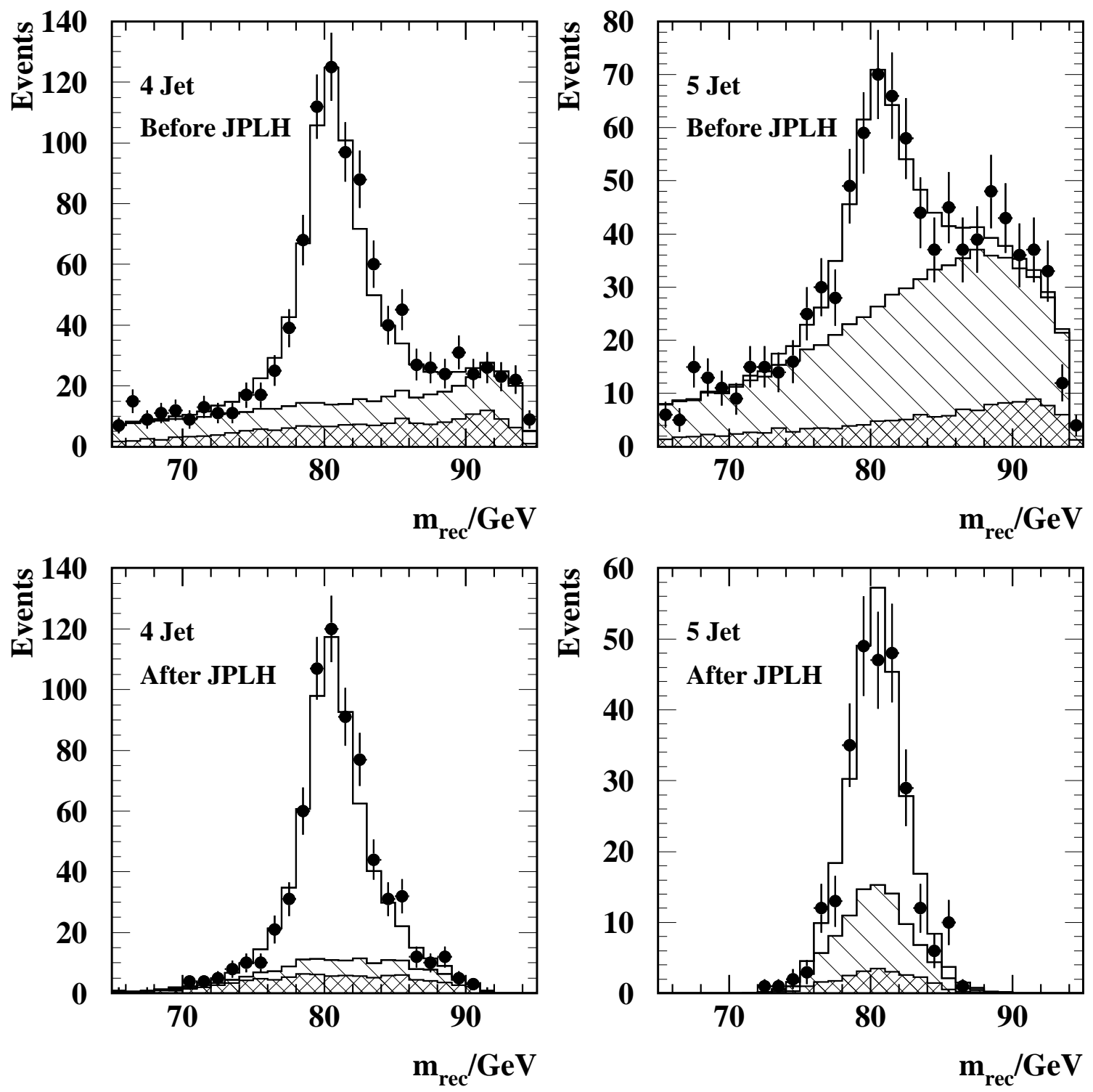

Figure 1: The reconstructed mass, $m_{\text {rec }}$, distributions for $\mathrm{W}^{+} \mathrm{W}^{-} \rightarrow$ qqqq events divided into four and five jet samples. The top two plots show the distributions before the jet-pairing likelihood (JPLH) cut. The lower two plots show the mass distributions which are used to determine $M_{\mathrm{W}}$, i.e. after the cut on JPLH. The points correspond to the OPAL data and the histograms to the Monte Carlo predictions. The contribution from the non-WW background is shown as the cross-hatched histogram and the combinatorial background is indicated by the singly-hatched histogram. 


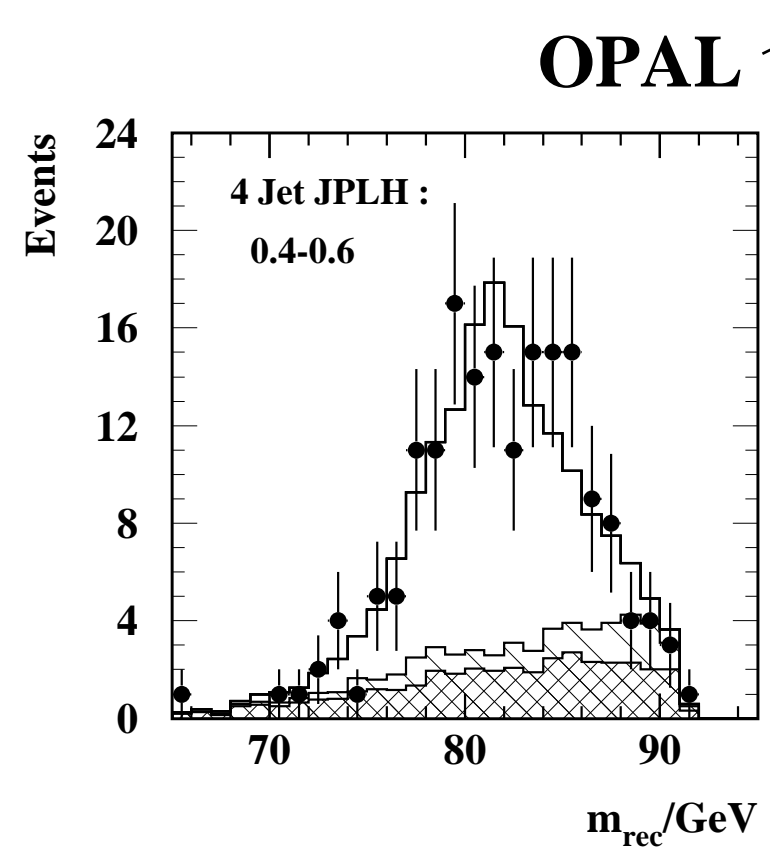

$V_{\mathbf{s}}=189 \mathrm{GeV}$
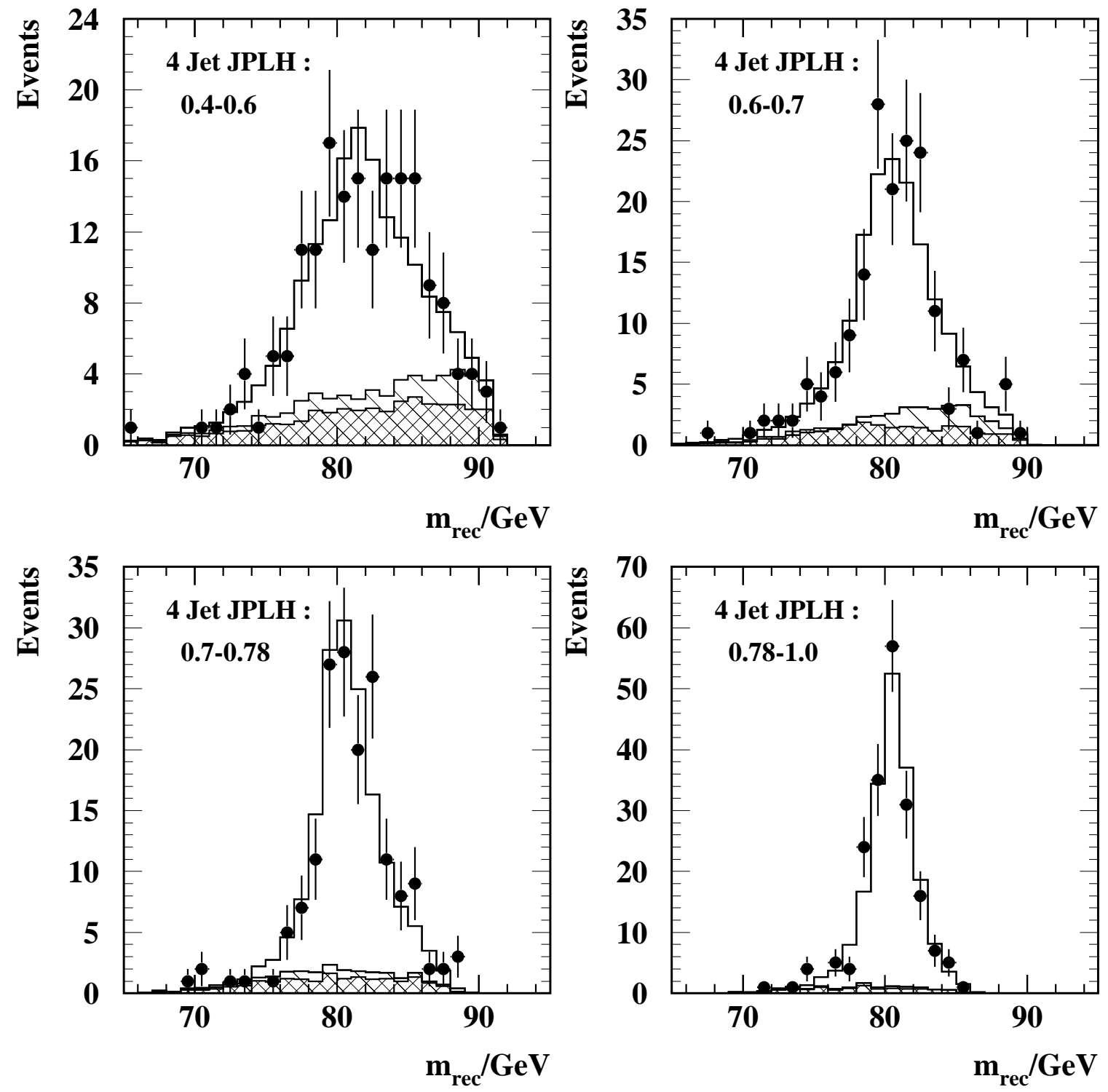

Figure 2: The reconstructed mass distributions for the four jet $\mathrm{W}^{+} \mathrm{W}^{-} \rightarrow$ qqqq sample divided into four bins of the output of the jet-pairing likelihood (JPLH) discriminant. The points correspond to the OPAL data and the histograms to the Monte Carlo predictions. The contribution from the non-WW background is shown as the cross-hatched histogram and the combinatorial background is indicated by the singly-hatched histogram. 

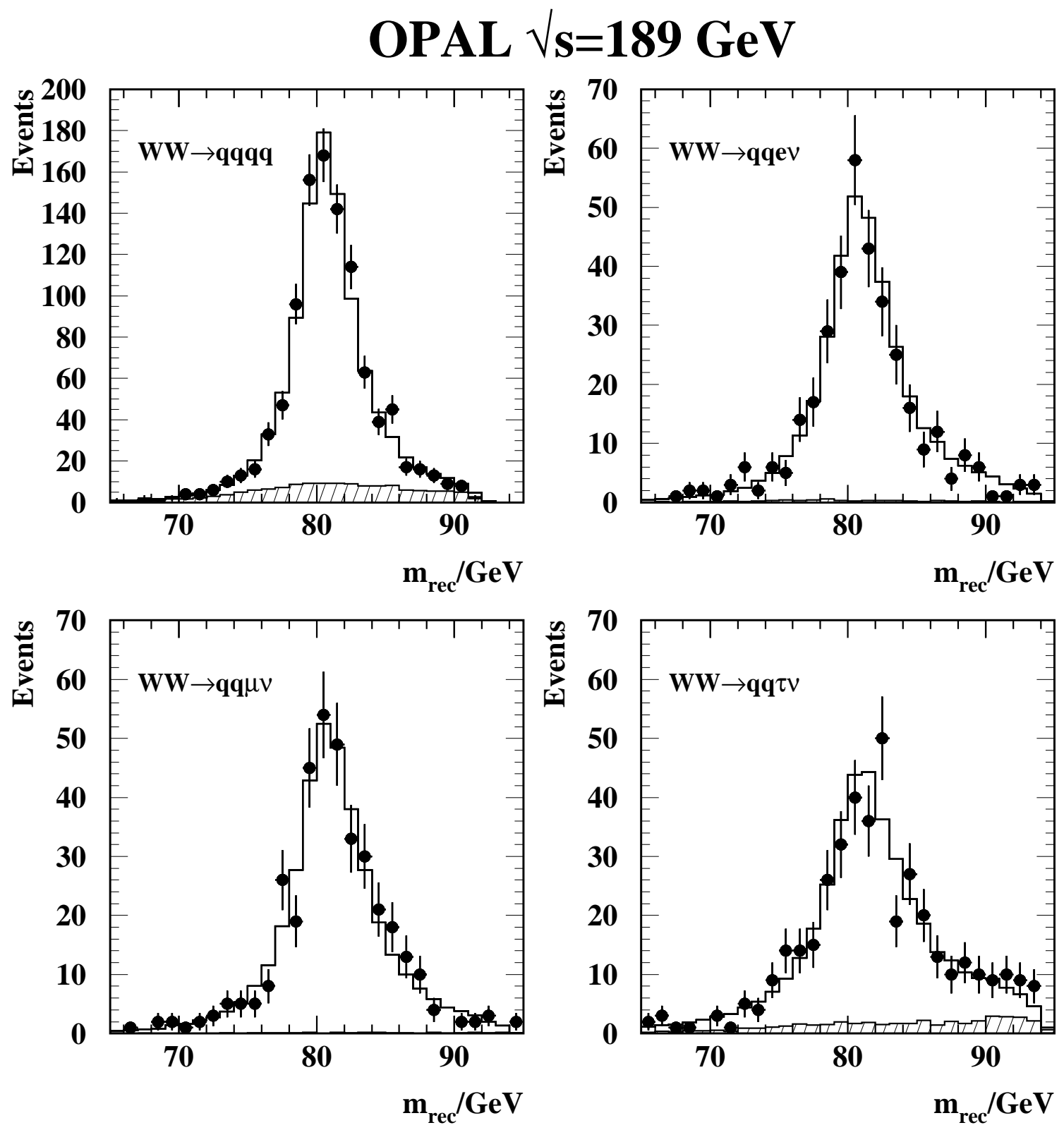

Figure 3: The reconstructed invariant mass distribution for the $\mathrm{W}^{+} \mathrm{W}^{-} \rightarrow \mathrm{qqqq}, \mathrm{W}^{+} \mathrm{W}^{-} \rightarrow \mathrm{qqe} \nu_{\mathrm{e}}$, $\mathrm{W}^{+} \mathrm{W}^{-} \rightarrow \mathrm{qq} \mu \nu_{\mu}$ and $\mathrm{W}^{+} \mathrm{W}^{-} \rightarrow \mathrm{qq} \tau \nu_{\tau}$ samples. The points correspond to the OPAL data and the histograms to the reweighted Monte Carlo spectra corresponding to the fitted masses. The non-WW background contribution is indicated by the hatched histogram. 


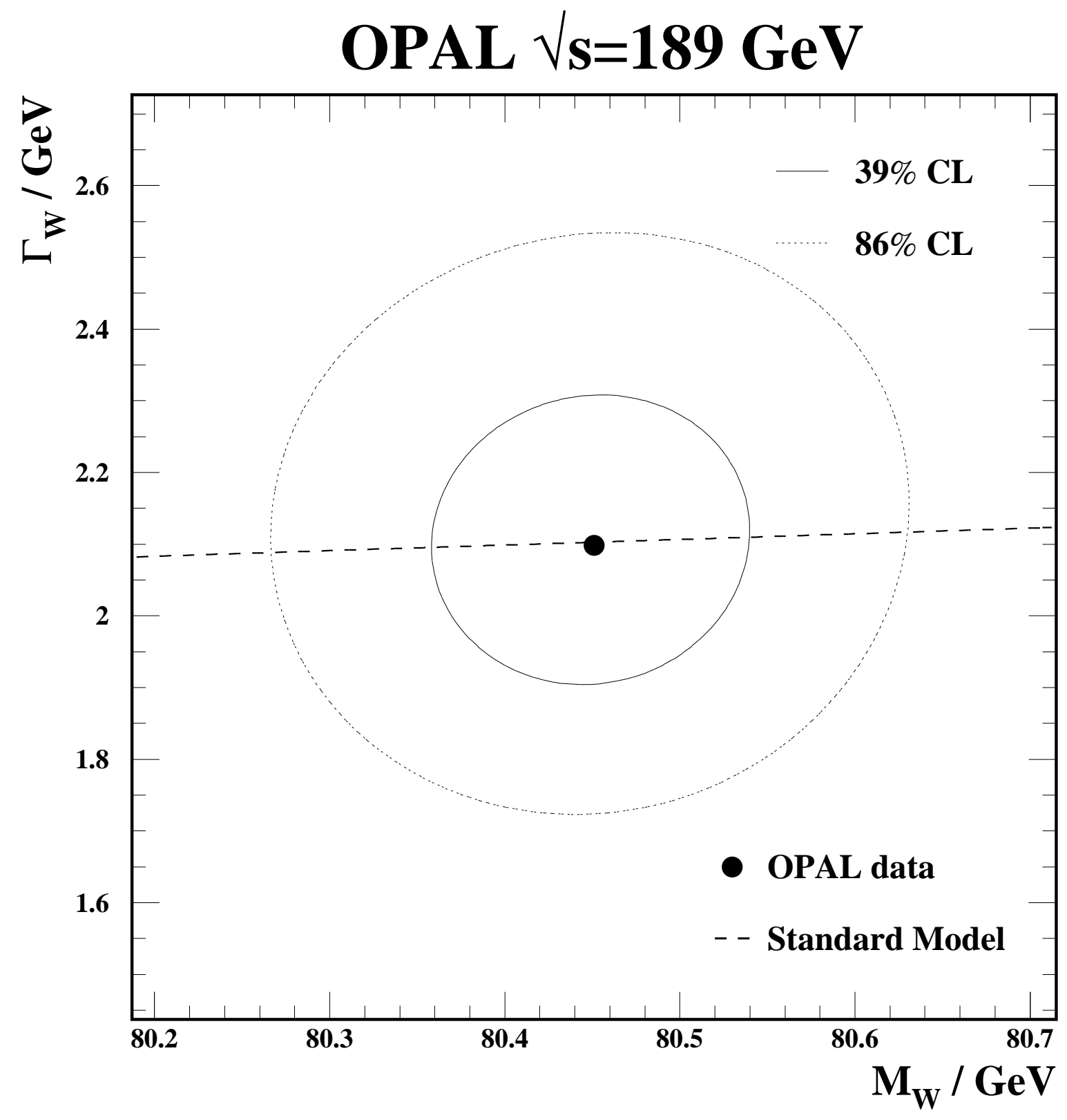

Figure 4: The 39\% and $86 \%$ contour levels of the two parameter fit using the reweighting method including systematic contributions. The Standard Model relation between $M_{\mathrm{W}}$ and $\Gamma_{\mathrm{W}}$, given in Equation 1, is shown by the dashed line. 